\title{
Navigating at night: fundamental limits on the sensitivity of radical pair magnetoreception under dim light
}

\author{
H. G. Hiscock, ${ }^{1,2}$ T. W. Hiscock, ${ }^{3,4}$ D. R. Kattnig,,${ }^{1,5}$ T. Scrivener, ${ }^{1}$ A. M. Lewis,,${ }^{1,6}$ \\ D. E. Manolopoulos, ${ }^{1}$ and P. J. Hore ${ }^{1, *}$
}

${ }^{1}$ Department of Chemistry, University of Oxford, Physical and Theoretical Chemistry Laboratory, Oxford OX1 3QZ, UK.

${ }^{2}$ Present address: Department of Chemistry, Massachusetts Institute of Technology, Cambridge, Massachusetts 02139, USA.

${ }^{3}$ Wellcome Trust/Cancer Research UK Gurdon Institute, University of Cambridge, Cambridge, UK.

${ }^{4}$ Cancer Research UK Cambridge Institute, University of Cambridge, Cambridge, UK.

${ }^{5}$ Permanent address: Living Systems Institute and Department of Physics, University of Exeter, Exeter EX4 4QD, UK.

${ }^{6}$ Present address: Department of Chemistry, University of Chicago, 5735 S Ellis Ave, Chicago, IL 60637, USA.

*Author for correspondence: peter.hore@chem.ox.ac.uk

Word count: 5540

Running title: Radical pair magnetoreception

\begin{abstract}
Night-migratory songbirds appear to sense the direction of the Earth's magnetic field via radical pair intermediates formed photochemically in cryptochrome flavoproteins contained in photoreceptor cells in their retinas. It is an open question whether this light-dependent mechanism could be sufficiently sensitive given the low light levels experienced by nocturnal migrants. The scarcity of available photons results in significant uncertainty in the signal generated by the magnetoreceptors distributed around the retina. Here we use results from Information Theory to obtain a lower bound estimate of the precision with which a bird could orient itself using only geomagnetic cues. Our approach bypasses the current lack of knowledge about magnetic signal transduction and processing in vivo by computing the best-case compass precision under conditions where photons are in short supply. We use this method to assess the performance of three plausible cryptochrome-derived flavin-containing radical pairs as potential magnetoreceptors.
\end{abstract}

Keywords: Cryptochrome. Information Theory. Light-dependent Magnetoreception. Nightmigratory songbirds. Radical Pair Mechanism. Spin Dynamics. 


\section{Introduction}

Night-migratory songbirds have a light-dependent magnetic compass that extracts directional information from the geomagnetic field (Cochran et al., 2004; Wiltschko and Wiltschko, 1995; Wiltschko, 1968; Wiltschko and Wiltschko, 1972). Signals from the primary receptors located in both eyes pass along the thalamofugal visual pathway to a small part of the forebrain known as Cluster $\mathrm{N}$ and are subsequently integrated with information from other orientation cues (Heyers et al., 2007; Mouritsen et al., 2005; Zapka et al., 2009; Zapka et al., 2010). The evidence (reviewed in (Hore and Mouritsen, 2016; Mouritsen, 2018)) points to a chemical sensing mechanism based on the quantum spin dynamics of photo-generated radical pairs in cryptochrome flavoproteins contained in photoreceptor cells in the birds' retinas (Hore and Mouritsen, 2016). Laboratory studies of the purified proteins suggest that cryptochromes have many of the properties required of a magnetic compass sensor (Kattnig et al., 2016; Maeda et al., 2012; Sheppard et al., 2017).

Although the cryptochrome hypothesis has received both experimental and theoretical support (Hore and Mouritsen, 2016), many aspects remain unclear. The notion that a sensory system makes use of quantum coherence in the "warm, wet and noisy" environment of a living cell has undoubtedly contributed to its popularity, at least amongst physicists (Al-Khalili and McFadden, 2014; Huelga and Plenio, 2013; Lambert et al., 2013; Marais et al., 2018; Mohseni et al., 2014), although the realization of such a mechanism in vivo is far from clear. Here we consider one of the many unresolved questions (Mouritsen, 2018): how could a light-dependent compass function efficiently under the dim light conditions experienced by nocturnal migrants (Hore and Mouritsen, 2016)? Specifically, we analyse the impact of the inherent uncertainty in the signal on the angular precision of the magnetic compass signal when the number of photoexcited magnetoreceptor molecules is limited by low photon flux.

Magnetic interactions many orders of magnitude weaker than the thermal energy $k_{\mathrm{B}} T$ are able to influence the reactivity of organic radical pairs if they are formed in a long-lived nonequilibrium electron-spin-coherent state (Maeda et al., 2008; Steiner and Ulrich, 1989). When, for example, a photo-excited singlet-state molecule accepts an electron from a closed-shell donor, a radical pair is formed in a non-stationary electronic singlet (S) state. This state interconverts coherently with the corresponding triplet state $(\mathrm{T})$ as a result of symmetrybreaking electron-nuclear hyperfine interactions. At the same time, the $\mathrm{S}$ and $\mathrm{T}$ states in this model undergo parallel spin-conserving reactions to form chemically distinct reaction products (see Fig. 1). The Zeeman interaction of the electron spins with an external magnetic field modifies the spin-eigenstates, alters the extent and timing of $\mathrm{S} \leftrightarrow \mathrm{T}$ interconversion, and so changes the branching ratio for the two competing reaction steps. The key quantities here are the ensemble-averaged fractional yields of the two products, $\Phi_{\mathrm{S}}$ and $\Phi_{\mathrm{T}}$ (with $\Phi_{\mathrm{S}}+\Phi_{\mathrm{T}}=1$ ). The anisotropy of the hyperfine interactions causes $\Phi_{\mathrm{S}}$ and $\Phi_{\mathrm{T}}$ to depend on the direction of 
the external magnetic field relative to the radicals in the retina, which are assumed to be immobilized and aligned. One of the two reaction products is presumed to act as the signalling state whose yield encodes information on which the bird could base navigational decisions.

Photo-induced electron transfer reactions in cryptochromes produce radical pairs in which the two electron spins are in a singlet state as a consequence of spin conservation (Cai et al., 2010; Gauger et al., 2011; Hogben et al., 2012). The nuclear spins, however, are assumed to be in thermal equilibrium when the radicals are first formed. Quantum mechanical simulations of magnetic field effects on the spin dynamics of radical pairs generally use density matrix methods to perform an average over the statistical ensemble of possible initial electron-nuclear spin states. The reaction kinetics (Fig. 1) are conventionally modelled using Haberkorn operators to capture the average decrease in the population of the radical pair state due to spinselective recombination (Haberkorn, 1976). By contrast, each individual radical pair will have a random initial configuration of its nuclear spins and will react at a different instant of time to give one of the two products. Fig. 2 gives an illustrative example of the singlet probability, $P_{\mathrm{S}}^{\alpha}\left(t_{\mathrm{R}}\right)$, (the probability of forming the singlet product if a reaction occurs at time $t_{\mathrm{R}}$ ) for two initial states of a simple radical pair. The average singlet reaction yield $\Phi_{\mathrm{S}}$ could be calculated by averaging over a large number of single-radical-pair reactions, propagating a random initial singlet state over time before a reaction event, at which point either the singlet or triplet product is formed. However, this would be exceedingly computationally expensive for all but the simplest radical pairs. Instead it would be better to derive the statistical distribution that describes the singlet reaction yield $\Phi_{\mathrm{S}}(N)$ given a finite number $N$ of photons each of which initiates a single radical pair reaction. In this way, the calculation would reduce to drawing random numbers from the appropriate distribution.

Having obtained the distribution of the singlet yield for a finite $N$ (Section II), we use Information Theory to circumvent the problem that next to nothing is known about how the singlet yield of cryptochromes spread around the retina is transduced, processed and interpreted (Section III). Use of the data processing inequality (Beaudry and Renner, 2011; Nielsen and Chuang, 2010) leads to a lower bound estimate of the precision with which a bird could orient itself using only the geomagnetic field as a directional cue (Section IV). This approach avoids the difficulty faced by a simpler Fisher information treatment (Guo et al., 2017; Lehmann and Casella, 1998; Vitalis and Kominis, 2017) which cannot account for arbitrary signal processing. We then estimate the photon flux into model cryptochrome-containing cells within the retina under the dim light conditions experienced by night-migratory songbirds (Section V) and assess the achievability of the Information Theory lower bound using a simple parametrized model of the singlet yield and two elementary data-processing approaches (Section VI). 
Finally, we analyse the performance of three plausible cryptochrome-derived radical pairs as potential magnetoreceptors.

\section{Distribution of reaction yields}

To derive the distribution of singlet yields for a finite number of single-radical-pair reactions, we first consider the case where $N=1$. The outcome of this reaction depends on the singlet probability, $P_{\mathrm{S}}^{\alpha}\left(t_{\mathrm{R}}\right)$, of the radical pair at the time when it reacts, $t_{\mathrm{R}}$, given the initial nuclear spin configuration, labelled $\alpha$. The probability that this radical pair forms the singlet product is just

$$
P\left(\Phi_{\mathrm{S}}(1)=1 \mid t_{\mathrm{R}}, \alpha\right)=P_{\mathrm{S}}^{\alpha}\left(t_{\mathrm{R}}\right) \equiv\left\langle\Psi^{\alpha}\left(t_{\mathrm{R}}\right)\left|\hat{P}_{\mathrm{S}}\right| \Psi^{\alpha}\left(t_{\mathrm{R}}\right)\right\rangle
$$

where $\hat{P}_{\mathrm{S}}$ is the singlet projection operator and $\left|\Psi^{\alpha}\left(t_{\mathrm{R}}\right)\right\rangle$ is the state of the radical pair at time $t_{\mathrm{R}}$ given an initial nuclear spin configuration $\alpha$. In order to compute the overall probability of forming the singlet product, we need to average over $t_{\mathrm{R}}$ and $\alpha$ by combining Equation (1) with the probabilities that the radical pair reacts at time $t_{\mathrm{R}}, p\left(t_{\mathrm{R}}\right)$, and has initial state $\alpha, P(\alpha)$. Assuming there is no nuclear polarization or coherence prior at the moment of formation of the radical pair, $P(\alpha)=1 / Z$ where $Z$ is the number of nuclear spin configurations. For symmetric recombination (i.e. $k_{\mathrm{S}}=k_{\mathrm{T}}=k$ in Fig. 1), $p\left(t_{\mathrm{R}}\right)=k \mathrm{e}^{-k t_{\mathrm{R}}}$ (Timmel et al., 1998).

Integrating this combined probability over all possible combinations of $t_{\mathrm{R}}$ and $\alpha$ (i.e. using the law of total probability) gives the overall probability that a single radical pair forms the singlet product:

$$
\begin{aligned}
P\left(\Phi_{\mathrm{S}}(1)=1\right) & =\sum_{\alpha} \int_{0}^{\infty} P\left(\Phi_{\mathrm{S}}(1)=1 \mid t_{\mathrm{R}}, \alpha\right) P(\alpha) p\left(t_{\mathrm{R}}\right) \mathrm{d} t_{\mathrm{R}} \\
& =\sum_{\alpha} \int_{0}^{\infty} P_{\mathrm{S}}^{\alpha}\left(t_{\mathrm{R}}\right) P(\alpha) p\left(t_{\mathrm{R}}\right) \mathrm{d} t_{\mathrm{R}} \\
& =E_{\alpha, t_{\mathrm{R}}}\left[P_{\mathrm{S}}^{\alpha}\left(t_{\mathrm{R}}\right)\right]=\Phi_{\mathrm{S}} .
\end{aligned}
$$

The probability that a single reaction results in the singlet product is simply the expectation value of the singlet probability of the radical pair, which is exactly the average singlet yield, $\Phi_{\mathrm{S}}$.

Using this result, we can now derive the distribution that describes the singlet yield for a finite number of reactions, $\Phi_{\mathrm{S}}(N)$. For $N$ reactions, because each reaction is independent and identically distributed, we have $N$ identical trials, each with probability $\Phi_{\mathrm{S}}$. The probability 
that $N \phi_{\mathrm{S}}$ of these reactions result in the singlet product is described by the binomial distribution $\mathcal{B}\left(N, \Phi_{\mathrm{S}}\right)$ (normalised by $\left.1 / N\right)$ :

$$
\Phi_{\mathrm{S}}(N) \sim \frac{1}{N} \mathcal{B}\left(N, \Phi_{\mathrm{S}}\right) \Rightarrow P\left(\Phi_{\mathrm{S}}(N)=\phi_{\mathrm{S}}\right)=\left(\begin{array}{c}
N \\
N \phi_{\mathrm{S}}
\end{array}\right) \Phi_{\mathrm{S}}^{N \phi_{\mathrm{S}}}\left(1-\Phi_{\mathrm{S}}\right)^{N-N \phi_{\mathrm{S}}}
$$

For large $N$ (the regime used exclusively below), the central limit theorem states that this binomial distribution is accurately approximated by a normal distribution $\mathcal{N}\left(\mu, \sigma^{2}\right)$ with mean $\mu=\Phi_{\mathrm{S}}$ and variance $\sigma^{2}$ :

$$
\begin{aligned}
& \Phi_{\mathrm{S}}(N) \sim \mathcal{N}\left(\Phi_{\mathrm{S}}, \Phi_{\mathrm{S}}\left(1-\Phi_{\mathrm{S}}\right) / N\right) \\
& \quad \Rightarrow p\left(\Phi_{\mathrm{S}}(N)=\frac{X}{N}\right) \approx\left(2 \pi \sigma^{2}\right)^{-1 / 2} \exp \left[-\frac{\left(\Phi_{\mathrm{S}}-X / N\right)^{2}}{2 \sigma^{2}}\right]
\end{aligned}
$$

The width of this distribution is $\sigma=\sqrt{\Phi_{\mathrm{S}}\left(1-\Phi_{\mathrm{S}}\right)} / \sqrt{N}$. For $p$ not close to 0 or $1, \sqrt{p(1-p)}$ does not depend strongly on $p$ and the majority of the uncertainty in $\Phi_{\mathrm{S}}(N)$ is determined by the number of reactions, and scales as $1 / \sqrt{N}$. Equation (4) is verified in the Supplementary Information, Section I using a Kinetic Monte Carlo algorithm (Voter, 2007).

\section{Information Theory}

Now that we can calculate the uncertainty in the singlet yield for a finite number of reactions, we can ask how efficiently a cryptochrome-based radical pair compass sensor would perform under low light conditions. The magnetoreceptors are located in the retinas of both eyes, probably in photoreceptor cells (Bolte et al., 2016; Günther et al., 2018; Hore and Mouritsen, 2016; Liedvogel and Mouritsen, 2010; Mouritsen et al., 2004; Niessner et al., 2011; Niessner et al., 2016). In the following, each cell is assumed to contain multiple, identically aligned cryptochrome molecules (Lau et al., 2012). The output of a cell would be an average of the singlet yields of all reaction cycles of its cryptochromes during a specified integration time. For a given orientation of the bird's head in the geomagnetic field (the "head direction", $\theta$ ), the curvature of the retina means that cryptochromes contained in cells at different positions within the retina will experience different magnetic field vectors, and so have different average singlet yields, $\Phi_{\mathrm{S}}$. Thus, information on the head direction is encoded in $\Phi_{\mathrm{S}}(\Theta ; N)=\left\{\Phi_{\mathrm{S}}^{i}(\Theta ; N)\right\}$, the set of singlet yields from all of the cells (labelled $i$ ) spread across the retina (Ritz et al., 2000). In the following we use upper case to denote random variables, e.g. $\Theta$, and lower case $(\theta)$ to denote sample values taken from the set of possible elements of those random variables. 
Unfortunately, we know so little about how the yield of the cryptochrome signalling state might be transduced, processed and interpreted (Dodson et al., 2013) that it is very difficult to reach conclusions about the precision of the compass without making assumptions of dubious validity. To circumvent this difficulty, we use a Rate Distortion Theory approach (Hilfinger et al., 2016; Lestas et al., 2010) to calculate a best-case compass precision for any given radical pair.

According to the title of Shannon's seminal paper (Shannon, 1948), the field of Information Theory (Cover and Thomas, 2012; Ihara, 1993; Jaynes, 1957), provides "a mathematical theory of communication" which encompasses the fundamental topics of signal processing and data compression. Rate Distortion Theory is specifically the analysis of lossy data compression. The simplest model involves an encoder-decoder pair in which the input data is compressed (generally to afford efficient communication) and then reconstructed by the decoder to give an output. The finite amount of information in the input data means that there is some limit on the extent to which the data can be compressed in order that it can be perfectly reconstructed by the decoder. Otherwise the encoding involves some loss of information that cannot be undone by the decoder, and results in the output being an imperfect copy of the input. In this case, Rate Distortion Theory allows one to calculate the expected error or difference between the input and the output.

Returning to the radical pair model, we can think of the external field direction (relative to the bird's head direction) as the source of data, which is encoded in the singlet yield of cryptochromes in receptor cells in the retina. This, in turn, is decoded by some unknown signal processing pathway to give an estimate of the head direction. If the available singlet yield signal is noisy due to a small photon flux, then the "compression" of the magnetic information will entail some error in the bird's estimation of its head direction, which we can quantify using several general inequalities from Rate Distortion Theory. A schematic representation of this process is given in Fig. 3.

The key quantities in information theory are the differential entropy, $h(X)$, which measures the uncertainty in the value of a random variable, $X$, based on its domain of possible values, and the conditional entropy $h(X \mid Y)$ which measures this uncertainty when the domain of $X$ is restricted by knowledge of $Y$. These quantities are defined in terms of the probability density functions $p(x)$ and $p(x, y)$ as (Shannon, 1948)

$$
h(X)=-\int p(x) \ln p(x) \mathrm{d} x,
$$




$$
h(X \mid Y)=-\iint p(x, y) \ln p(x \mid y) \mathrm{d} x \mathrm{~d} y
$$

Given the mean $(\mu)$ and variance $\left(\epsilon^{2}\right)$ of the probability density of $X$, an important result is that the entropy is maximal when the distribution of $X$ is Gaussian (Cover and Thomas, 2012), i.e. when $X \sim \mathcal{N}\left(\mu, \epsilon^{2}\right)=\left(2 \pi \epsilon^{2}\right)^{-1 / 2} \exp \left[-(x-\mu)^{2} / 2 \epsilon^{2}\right]$. Using Equation (5), the entropy of $X$ therefore satisfies

$$
h(X) \leq h\left(X \sim \mathcal{N}\left(\mu, \epsilon^{2}\right)\right)=\frac{1}{2} \ln \left(2 \pi \mathrm{e} \epsilon^{2}\right) .
$$

$h(X)$ and $h(X \mid Y)$ can be combined to compute the "mutual information" about variable $X$ available from a stochastic source of data $Y$ (Shannon, 1948):

$$
I(X ; Y) \equiv h(X)-h(X \mid Y)
$$

The narrower the distribution of $X$ when conditioned by $Y$, the lower the conditional entropy $h(X \mid Y)$ and so the larger the mutual information (Cover and Thomas, 2012). If $X$ is entirely determined by $Y$, the information is maximal and is determined by the entropy of $X$. If, instead, $X$ and $Y$ are independent, $h(X \mid Y)=h(X)$ and the mutual information is zero. Equation (8) is the basis of our derivation of a lower bound on the error in the head direction derived from the radical pair sensors.

Returning to our application, we are interested in how precisely the bird can estimate its head direction, $\Theta$, which determines the orientation of the geomagnetic field experienced by the magnetoreceptors in the retina. We assume that the system represents a Markov chain $\Theta \rightarrow \Phi_{S} \rightarrow \hat{\Theta}$ in which the bird's estimate of its head direction, $\hat{\Theta}$, is based solely on the encoding of the geomagnetic field direction in the singlet yield data coming from magnetoreceptor cells distributed across the retina, $\Phi_{\mathrm{S}}(\Theta ; N)$. Our aim is to quantify the mutual information between the two ends of the signalling pathway, $I(\Theta ; \hat{\Theta})$, represented by panels A and E in Fig. 3.

If the number of reactions $N=0$ there is clearly no information available about the external magnetic field. $\Theta$ and $\hat{\Theta}$ are therefore independent and the mutual information is zero. As $N$ increases, the amount of information available from the singlet yield signal increases as the signal-to-noise ratio improves (the noise scales as $1 / \sqrt{N}$ ). In the limit $N \rightarrow \infty$ (assuming noiseless signal transduction), the information about the magnetic field is perfectly encoded in $\Phi_{\mathrm{S}}(\Theta ; N)$, so that the conditional entropy $h(\Theta \mid \hat{\Theta})$ is minimised and $I(\Theta ; \hat{\Theta})$ is maximal, meaning that the head direction can be determined exactly. 
However, as we do not know how $\Phi_{\mathrm{S}}(\Theta ; N)$ is quantified, transmitted and decoded in the eye and the brain, we cannot quantify $h(\Theta \mid \hat{\Theta})$ and therefore cannot calculate the mutual information $I(\Theta ; \hat{\Theta})$. To sidestep this difficulty, we use a bound on this mutual information applicable for Markov chains - the data processing inequality (Beaudry and Renner, 2011; Nielsen and Chuang, 2010),

$$
I(\Theta ; \hat{\Theta}) \leq I\left(\Theta ; \Phi_{\mathrm{S}}(\Theta ; N)\right)
$$

which states that the mutual information between $\Theta$ and $\hat{\Theta}$ is bounded by $I\left(\Theta ; \Phi_{\mathrm{S}}(\Theta ; N)\right)$. Because the estimate of the head direction is downstream of the singlet yield signal, and because $\hat{\Theta}$ depends on $\Theta$ only via the singlet yield profile, any degradation in the input cannot be undone by decoding $\Phi_{\mathrm{S}}(\Theta ; N)$. The significance of this is that, as we can compute the mutual information between $\Theta$ and $\Phi_{\mathrm{S}}(\Theta ; N)$, we can compute an upper bound on the information between $\Theta$ and $\hat{\Theta}$, for any arbitrary decoding of the singlet yield signal to compute $\hat{\Theta}$. A simpler approach in which the Cramér-Rao lower bound is obtained from the Fisher information is inappropriate here because assumptions about the signal processing would be required to relate $\Phi_{\mathrm{S}}(\Theta ; N)$ to $\hat{\Theta}$ (Cramér, 1999; Lehmann and Casella, 1998).

In the following section, we use Equation (9) together with well-known results from Information Theory (Cover and Thomas, 2012; Ihara, 1993; Jaynes, 1957) to compute a lower bound estimate of the mean-squared difference between $\Theta$ and $\hat{\Theta}$ in order to evaluate how the precision of the compass sensor depends on $N$ and the properties of the radical pair.

\section{Lower bound error in the head direction}

We start with the mutual information between $\Theta$ and $\hat{\Theta}$ (Equation (8)):

$$
I(\Theta ; \hat{\Theta}) \equiv h(\Theta)-h(\Theta \mid \hat{\Theta})
$$

The bird can face in any direction with equal probability, i.e. the head direction is uniformly distributed, so that Equation (5) gives

$$
h(\Theta)=-\int_{-\pi}^{\pi}\left(\frac{1}{2 \pi}\right) \ln \left(\frac{1}{2 \pi}\right) \mathrm{d} \theta=\ln (2 \pi) .
$$

The mutual information, Equation (10), can be related to $h(\Theta-\hat{\Theta})$ using

$$
h(\Theta \mid \hat{\Theta})=h(\Theta-\hat{\Theta} \mid \hat{\Theta}) \leq h(\Theta-\hat{\Theta})
$$


The equality in Equation (12) arises because we are in effect subtracting a constant in the conditional entropy, and Shannon differential entropy is invariant under translation (Cover and Thomas, 2012). The inequality is Shannon's lower bound which comes from the well-known concept that conditioning cannot increase entropy (Shannon, 1959).

Using Equation (7), we can evaluate a bound on $h(\Theta-\hat{\Theta})$ in terms of the variance $\epsilon^{2}$ of the distribution of $\Theta-\hat{\Theta}$ :

$$
\epsilon^{2} \geq \frac{1}{2 \pi \mathrm{e}} \exp (2 h(\Theta-\hat{\Theta}))
$$

Since the expectation value $E[\Theta-\hat{\Theta}]=0, \epsilon^{2}$ is the mean-squared difference between $\Theta$ and $\hat{\Theta}$ :

$$
\epsilon^{2}=E\left[\{(\Theta-\hat{\Theta})-E[\Theta-\hat{\Theta}]\}^{2}\right]=E\left[(\Theta-\hat{\Theta})^{2}\right]
$$

Combining Equations (9) to (13) gives an expression for the lower bound, $\epsilon_{\mathrm{LB}}$, the root-meansquared error in the head direction estimate based on the encoding of the true head direction in the singlet yield signal across the retina:

$$
\epsilon^{2} \geq \epsilon_{\mathrm{LB}}^{2}=\frac{2 \pi}{\mathrm{e}} \exp \left[-2 I\left(\Theta ; \Phi_{\mathrm{S}}(\Theta ; N)\right)\right]
$$

The real power of this method is that Equation (15) gives the lower bound of $\epsilon$ in the limit that $100 \%$ of the information available in the singlet yield signal is retained. This is valid for any arbitrarily sophisticated data processing algorithm used to interpret the singlet yield signal, and so successfully avoids having to make any guesses about signal transduction and processing in vivo, instead assuming the optimal case. Considering the complexity of data processing known in, for instance, the visual system (Hubel, 1995), the ability to account for a highly developed but unknown decoding mechanism is an important one. The evaluation of $\epsilon_{\mathrm{LB}}$ is detailed in the Supplementary Information, Section II.

\section{Magnetoreceptor cells}

The next stage is to define the orientations of the magnetoreceptor molecules in the retina with respect to the geomagnetic field. We model a bird's eye as a sphere with the retina spanning a hemispherical section of its surface and the pupil positioned on the optical axis opposite the centre of the retina. Although a crude approximation to reality, this geometry captures the 
essential physics. The cells that contain the magnetoreceptor molecules are assumed to be identical, uniformly arrayed across the retina, and cylindrically shaped (in the manner of the photoreceptor cells) with their symmetry axes pointing towards the centre of the sphere. Within each cell, all magnetoreceptor molecules, and therefore all radical pairs formed within them, are taken to have identical orientations. For simplicity, the optical axis of the eye is aligned parallel to the horizontal component of the geomagnetic field $(\theta=0)$. The geomagnetic inclination (with respect to the horizontal) is taken as $\phi=66^{\circ}$. In this model, therefore, the orientation of a radical pair with respect to the geomagnetic field vector is completely determined by the position in the retina of the cell in which it resides. We specify this position by co-latitude $(\kappa)$ and azimuth $(v)$ angles and, for simplicity, assume a geometry within each cell such that the angle, $\zeta$, between the magnetic field vector and the main axis of the radical pair is independent of the co-latitude and given by (see Supplementary Information, Section III)

$$
\cos \zeta=\sin (v) \sin (\phi)
$$

Evaluation of the minimum head direction error, Equation (15), requires the diagonalization of a covariance matrix (see Supplementary Information, Section II) whose dimension is the total number of magnetoreceptor cells in the retina (potentially $>10^{6}$ ). To make the problem tractable, we reduce the dimension of the system by grouping the cells contained in spherical caps of half-angle $\xi=0.5^{\circ}$ to form a smaller number of "grouped magnetoreceptor cells". The cells within each group have such similar positions and orientations that they will have almost identical average singlet yields, $\Phi_{\mathrm{S}}$ (Worster et al., 2017). Each group is treated as a single receptor cell that absorbs the total number of photons that would impinge on the constituent cells during the integration time. The retina is thus described by a square grid of these $1^{\circ}$ spaced grouped receptor cells. In the visual system, similar groupings of photoreceptor cells form under dim light conditions to improve contrast at the expense of resolution (Warrant, 1999). As the increase in the number of photons per receptor compensates the reduction in the number of receptor cells, we consider this simplification to be valid. To verify this, we calculated $\epsilon_{\mathrm{LB}}$ using $\xi=1.0^{\circ}$, representing a four-fold decrease in the number of grouped receptor cells each of which received four times as many photons. The calculated lower bound errors agreed to within a few percent, justifying the approximation.

The double-cone photoreceptor cells have been proposed as the most likely location of the magnetoreceptors (Günther et al., 2018; Worster et al., 2017). They are arranged on an approximately hexagonal lattice in the retina with a nearest neighbour separation of $7 \mu \mathrm{m}$ (Kram et al., 2010), giving an average of one double cone per $40 \mu \mathrm{m}^{2}$ of retina. Taking $r=4$ $\mathrm{mm}$ as the typical radius of the eye of a small songbird (Thomas et al., 2002), the area of a 
spherical cap with half-angle $\xi=0.5^{\circ}$ is $2 \pi r^{2}(1-\cos \xi)=3800 \mu \mathrm{m}^{2}$. There are therefore $\sim 100$ double cones in each grouped receptor cell and $\sim 30,000$ grouped receptor cells in the model retina.

We now need to estimate the number of photons, $N$, that enter each grouped receptor cell in a given time interval. Birds can orient using their magnetic compass on cloudless, moonless nights at light intensities down to $3 \times 10^{-4}$ lux corresponding to an average of $\sim 0.3$ photons incident on each $1 \mu \mathrm{m}^{2}$ of retina per second (Cochran et al., 2004; Hore and Mouritsen, 2016). A typical photoreceptor cell has diameter $\approx 2 \mu \mathrm{m}$ and therefore occupies $\sim 3 \mu^{2}$, meaning that each cell receives roughly 1 photon per second under these conditions. Since there are $\sim 100$ cells per grouped receptor cell, we have $N \approx 100$ photons per grouped receptor cell per second.

The signal-to-noise ratio of the sensor can be improved by increasing the period during which photons are absorbed and integrating the resulting signals (Warrant, 1999). Following Cochran et al. (Cochran et al., 2004), it seems unlikely that this integration period would be much longer than a few minutes, meaning that the number of photons per grouped receptor cell is in the range $10^{2} \leq N \leq 10^{4}$ when the light intensity is $\sim 3 \times 10^{-4}$ lux.

\section{A model radical pair}

The singlet yield signals produced by grouped receptor cells spread across the whole of the retina can be pictured as "visual modulation patterns" (Ritz et al., 2000) (VMP) in which the singlet yield for each grouped receptor cell is represented here by a colour-coded pixel in a redto-blue heat map. To obtain a two-dimensional pattern, the retina is projected onto a plane tangent to the centre of the retina using the pupil as the point of perspective. This stereographic projection maps the position of a grouped receptor cell onto a point in this plane defined by Cartesian coordinates $(x, y)$ :

$$
\begin{aligned}
\tan (\kappa / 2) & =\sqrt{x^{2}+y^{2}}, \\
\tan (v) & =x / y .
\end{aligned}
$$

The co-latitude and azimuth are thus represented, respectively, by radial and angular features in the VMP. The centre of the retina $(\kappa=0)$ projects to the centre $(x=y=0)$ of the VMP (Lau et al., 2012; Worster et al., 2017).

Before applying the Information Theory approach to realistic radical pairs, we begin with a simple parametrized model of the dependence of the average singlet yield on the direction, $\zeta$, of the geomagnetic field with respect to the radical pair (see Supplementary Information, Section III), 


$$
\Phi_{\mathrm{S}}=\frac{1}{2}-A \exp \left[-\frac{\left(\zeta-90^{\circ}\right)^{2}}{2 \sigma^{2}}\right]
$$

This form of $\Phi_{\mathrm{S}}$ is an inverted Gaussian of amplitude $A$, taken as $A=1.27 \times 10^{-3}$, and width $\sigma$, centred at $\zeta=90^{\circ}$, superimposed on a constant background of amplitude 0.5 . The value of $A$ was chosen to match that found in simulations of a 16-spin model of a cryptochrome-based radical pair with a lifetime, $\tau=1 / k=1 \mu$ s (Hiscock et al., 2016). In this simple example, the alignment of the radical pairs in the receptor cells is such that the signal is independent of the co-latitude of the cell (Equation (16)) and therefore has no radial variation in the VMP (Fig. 4d).

Fig. 4a gives an example of how the singlet yield signal across the retina varies for different values of $N$, when $\sigma=26^{\circ}$. As anticipated, the noise arising from the variance of $\Phi_{\mathrm{S}}^{i}(\Theta, N)$ tends to obscure the signal for the smaller numbers of photons per receptor cell, $N$. Each 100fold increase in $N$ improves the signal-to-noise ratio by a factor of $\sim 10$ (Equation (4)) and, by eye, the signal only emerges from the noise when $N \geq 10^{6}$.

Before considering the Information Theory lower bound, we assess its achievability using two simple data processing methods to compute the root-mean-squared error in the derived head direction. In the first, we average the noisy signal along the diameters of the VMP and obtain a crude estimate of the head direction, $\hat{\theta}$, as the value of the azimuth that corresponds to the minimum of this processed signal, e.g. Fig. $4 \mathrm{~b}$ (where $\hat{\theta} \approx 95^{\circ}$ ). This procedure was repeated for many realisations of the singlet yield signal, $\Phi_{\mathrm{S}}(\Theta ; N)$, so that the mean-squared error, $\epsilon^{2}=E\left[(\Theta-\hat{\Theta})^{2}\right]$ can be calculated. The second, more sophisticated, data processing method entails fitting a Gaussian model function to the diameter-averaged signals to obtain an improved estimate of the head direction from the value of the azimuth at the minimum of the fitted model function (Fig. 4b). Again, this is repeated many times, and the mean-squared error in the head direction computed. These calculations have been constructed such that both processing methods give the correct result when $N \rightarrow \infty$.

Fig. 4c compares the Information Theory lower bound, $\epsilon_{\mathrm{LB}}$, with the root-mean-squared error $\epsilon$ derived from the above two methods. In all three cases, the precision improves as $N$ increases, as expected. The curve-fitting method offers a relatively good proof of principle that the Information Theory bound is not much smaller than that achievable by data processing. Indeed, some more sophisticated processing method could achieve a better agreement, but the importance of the Information Theory result is that it provides a bound. The more primitive 
interpretation technique, based on selecting the minimum value of the diameter-averaged signals gives a far larger error.

The same model of the singlet yield (Equation (18)) can be used to assess how the width, $\sigma$, of the signal affects the precision of the compass (Fig. 4d). This is of interest in relation to recent simulations of cryptochrome-based radical pairs which predict that for lifetimes exceeding $\sim 1$ $\mu \mathrm{s}, \Phi_{\mathrm{S}}$ contains a sharp feature, referred to as a spike or "needle" (Hiscock et al., 2016), that could deliver a much more precise compass bearing than previously thought possible. Fig. $4 \mathrm{e}$ shows $\epsilon_{\mathrm{LB}}$ as a function of $\sigma$ for $N=100$ and 1000. When the number of photons is limited, there is an optimum width that minimises the error in the estimated head direction. The more photons there are, the narrower is the optimal width of $\Phi_{\mathrm{S}}$. It appears that for small $N$, more spatial averaging is required to extract directional information, and very narrow signals are less well suited to this coarse-graining.

\section{Cryptochrome-based radical pairs}

At least three radical pairs have been discussed as potential magnetic sensors in cryptochromes in vivo. $\left[\mathrm{FAD}^{\bullet-} \mathrm{TrpH}^{\bullet+}\right]$ is formed by light-induced electron transfer from a tryptophan $(\mathrm{TrpH})$ amino acid residue within the protein to the fully oxidised FAD (flavin adenine dinucleotide) chromophore and gives rise to magnetic field effects on long-lived states of the purified protein (Kattnig et al., 2016; Maeda et al., 2012; Sheppard et al., 2017). Alternatively, a radical pair could be formed during the re-oxidation of the fully (photochemically) reduced state by electron transfer from $\mathrm{FADH}^{-}$to an unknown electron acceptor (Niessner et al., 2014; Niessner et al., 2013). We consider two possibilities, both of which have been proposed previously: $\left[\mathrm{FAD}^{\bullet-} \mathrm{Z}^{\bullet}\right]$ in which $\mathrm{Z}^{\bullet}$ is a radical devoid of hyperfine interactions (Hogben et al., 2009; Müller and Ahmad, 2011; Ritz et al., 2009; Solov'yov and Schulten, 2009), and [FAD ${ }^{\bullet-}$ Asc $^{\bullet-}$ ], formed when ascorbic acid is the electron donor (Lee et al., 2014; Nielsen et al., 2017). Simulations suggest that both could produce much larger anisotropic magnetic field effects than $\left[\mathrm{FAD}^{--} \mathrm{TrpH}^{\bullet+}\right]$ and could therefore form the basis of a more sensitive direction sensor. With the hyperfine interactions used here (Supplementary Information, Section IV), only $\left[\mathrm{FAD}^{\bullet-} \mathrm{TrpH}^{\bullet+}\right]$ is predicted to exhibit the narrow spike mentioned above (Hiscock et al., 2016). In the following, we abbreviate these three radical-pair states of cryptochrome as FADTrp, FAD-Z and FAD-Asc. To compare their performance under low light conditions, we use the same intracellular orientation of the cryptochromes as in Fig. 4, any choice being arbitrary given our ignorance of the situation in vivo (see Supplementary Information, Section III). The dependence of $\Phi_{\mathrm{S}}$ on the direction of a $50 \mu \mathrm{T}$ magnetic field was calculated as described previously using the hyperfine interaction tensors listed in the Supplementary Information, Section IV. 
Fig. 5a shows the lower bound error for FAD-Trp with lifetimes of 1, 2 and $5 \mu \mathrm{s}$. As the lifetime increases, the singlet yield signal shows both an increase in anisotropy and the appearance of the compass "needle" (Hiscock et al., 2016) for $\tau \geq 5 \mu \mathrm{s}$. It is therefore not surprising that $\epsilon_{\mathrm{LB}}$ is smaller for the longer lived radical pairs. When $\tau$ for FAD-Trp is extended to $100 \mu \mathrm{s}$, the spike becomes much sharper and $\epsilon_{\mathrm{LB}} \approx 0$ for $N \geq 100$ (not shown). By contrast, FAD-Z, because of its much larger magnetic anisotropy, has $\epsilon_{\mathrm{LB}} \approx 0$ for $N \geq 100$ and $\tau \geq 1 \mu$ s (also not shown). For a given lifetime, FAD-Z offers a much more precise estimate of the head direction than does FAD-Trp.

The results shown in Fig. 5a were obtained using the previously studied 16-spin model of FADTrp (details in Supplementary Information, Section IV) (Lee et al., 2014), neglecting the exchange and dipolar interactions between the radicals. Both approximations greatly reduce the computational difficulty of the spin dynamics calculations but lead to overestimates of the anisotropy of $\Phi_{\mathrm{S}}$ (Lewis, 2018).

A calculation that includes all 27 hyperfine-coupled nuclear spins in FAD-Trp together with realistic exchange and dipolar interactions is not feasible using an exact quantum mechanical approach. However, Lewis (Lewis, 2018) has shown that approximate calculations (with an estimated 5\% statistical error) can be performed on spin systems of this size using the semiclassical algorithm (Manolopoulos and Hore, 2013). As shown in Table 1, there is a decrease in the anisotropy of the signal of over an order of magnitude in going from 14 nuclear spins to 27. As expected, this decrease is mirrored in the calculated lower bound error (Fig. $5 \mathrm{~b}$ ) which is large even for $N \geq 10^{5}$ photons per grouped receptor when $\tau=1 \mu \mathrm{s}$. This suggests that there may be a serious problem with a FAD-Trp-based compass sensor under low light conditions.

Finally, we turn to FAD-Asc and model a rapidly tumbling Asc ${ }^{\bullet-}$ radical as the partner of the $\mathrm{FAD}^{\bullet-}$ radical (details in Supplementary Information, Section IV). Compared to the 16-spin model of FAD-Trp, the singlet yield anisotropy is larger by a factor of about 50 when $\tau=1$ $\mu$ s. This again leads to a very small head direction error (Table 1), and suggests that an organic counter-radical with small hyperfine interactions could prove to be a more sensitive compass sensor in vivo. However, both FAD-Asc and FAD-Z are expected to show a similar decrease in anisotropy as noted above for FAD-Trp pair on inclusion of the radical-radical interactions.

\section{Conclusions}

We have investigated the functioning of a radical pair compass under the low-light conditions experienced by night-migratory songbirds and in particular how quantum noise could affect a bird's ability to estimate its head direction from the quantum yield of the signalling state of 
cryptochrome magnetoreceptors spread around the retina. Within the current model of the radical pair mechanism, our analysis represents a best-case scenario in a number of ways. We have assumed: (a) that the processing required to decode the information supplied by the retina is as efficient as it could possibly be; (b) that all the cryptochrome molecules in a cell are identically and perfectly aligned; (c) that the radical pairs undergo negligible spin relaxation; and (d) that all the light entering the eye is absorbed by cryptochromes despite the existence of the opsin proteins responsible for vision.

Subject to these limitations, two main conclusions emerge from this study. First, the low photon flux on a clear moonless night appears to pose a significant sensitivity challenge for a lightdependent magnetoreceptor, whatever the identity of the radical pair. Second, our analysis suggests that $\left[\mathrm{FAD}^{\bullet-} \mathrm{TrpH}^{\bullet+}\right]$ is less well suited as a sensor than a radical pair in which the tryptophan radical has been replaced by one containing no $\left(\left[\mathrm{FAD}^{\bullet-} \mathrm{Z}^{\bullet}\right]\right)$ or very few $\left(\left[\mathrm{FAD}^{\bullet-}\right.\right.$ $\mathrm{Asc}^{\bullet-}$ ) hyperfine couplings. Other things being equal, [FAD $\left.{ }^{\bullet-} \operatorname{TrpH}{ }^{\bullet+}\right]$ is expected to be much less sensitive to weak magnetic fields because of the large number of ${ }^{1} \mathrm{H}$ and ${ }^{14} \mathrm{~N}$ nuclei in $\mathrm{TrpH}^{\bullet+}$ which translates into an increased error in the derived compass bearing. However, it is at least clear that $\left[\mathrm{FAD}^{\bullet-} \mathrm{TrpH}^{\bullet+}\right]$ is formed photochemically in purified cryptochromes and that it generates magnetic field effects. The same cannot be said of the other two radical pairs whose existence, let along magnetic sensitivity, has not been demonstrated, even in vitro.

It must be stressed that throughout this study we have tacitly assumed that the current model of radical pair magnetoreception is appropriate and complete. If there were some aspect of the behaviour of radical pairs that made them, say, $10^{3}$ times more sensitive to the geomagnetic field, then the problems associated with low photon flux would be much less serious. Although such huge increases in detection sensitivity seem unlikely, they have been discussed before in the context of the extraordinary finding that migratory birds are unable to use their magnetic compass when exposed to extremely weak radiofrequency fields (Hiscock et al., 2017). Additional, and equally speculative, sources of large sensitivity gains include abnormally slow electron spin relaxation ( $\gtrsim 10 \mu$ s) (Hiscock et al., 2016), greatly extended signal-averaging times ( $\gtrsim 1 \mathrm{hr}$ ), and spin-selective radical scavenging reactions which, at least theoretically, could significantly enhance the magnetic sensitivity (Kattnig, 2017; Kattnig and Hore, 2017). There has also been some discussion, reviewed by (Hore and Mouritsen, 2016), of whether the electron spin entanglement with which singlet radical pairs are formed could be a source of additional sensitivity. Entanglement has no known function within the current model of radical pair magnetoreception and a radical pair formed in an unpolarised triplet state (which has no entanglement) could also be a viable magnetic compass sensor, suggesting that entanglement is not an essential requirement (Hogben et al., 2012). Finally, the non-uniform illumination of the retina produced by a starry sky could improve the signal-to-noise ratio in a subset of 
magnetoreceptors from which a more reliable estimate of the head direction could be obtained (Hore and Mouritsen, 2016).

\section{Financial support}

This work was supported by the European Research Council under the European Union's 7th Framework Programme, FP7/2007-2013/ERC, grant agreement no. 340451 (Advanced Grant: ChemNav) and the European Union's Horizon 2020 research and innovation programme, grant agreement no. 810002 (Synergy Grant: QuantumBirds), and the Air Force Office of Scientific Research (Air Force Materiel Command, USAF award no. FA9550-14-1-0095). T.W.H. is supported by an EMBO Long-Term Fellowship (ATLF 606-2018) and a Wellcome Strategic Award (105031/D/14/Z).

\section{Conflict of interest.}

None. 
Al-Khalili, J and Mcfadden, J. (2014). Life on the edge: the coming of age of quantum biology. London: Bantam Press.

Beaudry, NJ and Renner, R. (2011). An intuitive proof of the data processing inequality. arXiv:1107.0740.

Bolte, P, Bleibaum, F, Einwich, A, Günther, A, Liedvogel, M, Heyers, D, Depping, A, Wöhlbrand, L, Rabus, R, Janssen-Bienhold, U and Mouritsen, H. (2016). Localisation of the putative magnetoreceptive protein cryptochrome $1 \mathrm{~b}$ in the retinae of migratory birds and homing pigeons. Plos One, 11, e 0147819.

Cai, J, Guerreschi, GG and Briegel, HJ. (2010). Quantum control and entanglement in a chemical compass. Physical Review Letters, 104, 220502.

Cochran, WW, Mouritsen, H and Wikelski, M. (2004). Migrating songbirds recalibrate their magnetic compass daily from twilight cues. Science, 304, 405-408.

Cover, TM and Thomas, JA. (2012). Elements of information theory. Hoboken: John Wiley \& Sons.

Cramér, H. (1999). Mathematical methods of statistics. Princeton: Princeton University Press.

Dodson, CA, Hore, PJ and Wallace, MI. (2013). A radical sense of direction: signalling and mechanism in cryptochrome magnetoreception. Trends in Biochemical Sciences, 38, 435-446.

Gauger, EM, Rieper, E, Morton, JJL, Benjamin, SC and Vedral, V. (2011). Sustained quantum coherence and entanglement in the avian compass. Physical Review Letters, 106, 040503.

Günther, A, Einwich, A, Sjulstok, E, Feederle, R, Bolte, P, Koch, KW, Solov'yov, IA and Mouritsen, H. (2018). Double-cone localization and seasonal expression pattern suggest a role in magnetoreception for European robin cryptochrome 4. Current Biology, 28, 211-223.

Guo, LS, Xu, BM, Zou, J and Shao, B. (2017). Quantifying magnetic sensitivity of radical pair based compass by quantum fisher information. Sci. Rep., 7, 5826.

Haberkorn, R. (1976). Density matrix description of spin-selective radical pair reactions. Molecular Physics, 32, 1491-1493.

Heyers, D, Manns, M, Luksch, H, Güntürkün, O and Mouritsen, H. (2007). A visual pathway links brain structures active during magnetic compass orientation in migratory birds. Plos One, 2, e937.

Hilfinger, A, Norman, TM, Vinnicombe, G and Paulsson, J. (2016). Constraints on fluctuations in sparsely characterized biological systems. Physical Review Letters, 116, 058101.

Hiscock, HG, Mouritsen, H, Manolopoulos, DE and Hore, PJ. (2017). Disruption of magnetic compass orientation in migratory birds by radiofrequency electromagnetic fields. Biophysical Journal, 113, 1475-1484. 
Hiscock, HG, Worster, S, Kattnig, DR, Steers, C, Jin, Y, Manolopoulos, DE, Mouritsen, H and Hore, PJ. (2016). The quantum needle of the avian magnetic compass. Proceedings of the National Academy of Sciences, USA, 113, 4634-4639.

Hogben, HJ, Biskup, T and Hore, PJ. (2012). Entanglement and sources of magnetic anisotropy in radical pair-based avian magnetoreceptors. Physical Review Letters, 109, 220501.

Hogben, HJ, Efimova, O, Wagner-Rundell, N, Timmel, CR and Hore, PJ. (2009). Possible involvement of superoxide and dioxygen with cryptochrome in avian magnetoreception: origin of Zeeman resonances observed by in vivo EPR spectroscopy. Chemical Physics Letters, 480, 118-122.

Hore, PJ and Mouritsen, H. (2016). The radical pair mechanism of magnetoreception. Annu. Rev. Biophys., 45, 299-344.

Hubel, DH. (1995). Eye, brain and vision: W. H. Freeman.

Huelga, SF and Plenio, MB. (2013). Vibrations, quanta and biology. Contemp. Phys., 54, 181-207.

Ihara, S. (1993). Information theory for continuous systems. Singapore: World Scientific.

Jaynes, ET. (1957). Information theory and statistical mechanics. Physical Review, 106, 620-630.

Kattnig, DR. (2017). Radical-pair-based magnetoreception amplified by radical scavenging: resilience to spin relaxation. Journal of Physical Chemistry B, 121, 10215-10227.

Kattnig, DR, Evans, EW, Déjean, V, Dodson, CA, Wallace, MI, Mackenzie, SR, Timmel, CR and Hore, PJ. (2016). Chemical amplification of magnetic field effects relevant to avian magnetoreception. Nat. Chem., 8, 384-391.

Kattnig, DR and Hore, PJ. (2017). The sensitivity of a radical pair compass magnetoreceptor can be significantly amplified by radical scavengers. Sci. Rep., 7, 11640.

Kram, YA, Mantey, S and Corbo, JC. (2010). Avian cone photoreceptors tile the retina as five independent, self-organizing mosaics. Plos One, 5, e8992.

Lambert, N, Chen, YN, Cheng, YC, Li, CM, Chen, GY and Nori, F. (2013). Quantum biology. Nat. Phys., 9, 10-18.

Lau, JCS, Rodgers, CT and Hore, PJ. (2012). Compass magnetoreception in birds arising from photo-induced radical pairs in rotationally disordered cryptochromes. J. Roy. Soc. Interface, 9, 3329-3337.

Lee, AA, Lau, JCS, Hogben, HJ, Biskup, T, Kattnig, DR and Hore, PJ. (2014). Alternative radical pairs for cryptochrome-based magnetoreception. J. R. Soc. Interface, 11, 20131063.

Lehmann, EL and Casella, G. (1998). Theory of point estimation. New York: Springer-Verlag.

Lestas, I, Vinnicombe, G and Paulsson, J. (2010). Fundamental limits on the suppression of molecular fluctuations. Nature, 467, 174-178.

Lewis, A. (2018). Spin dynamics in radical pairs: Springer International Publishing.

Liedvogel, M and Mouritsen, H. (2010). Cryptochromes - a potential magnetoreceptor: what do we know and what do we want to know? J. R. Soc. Interface, 7, S147-S162. 
Maeda, K, Henbest, KB, Cintolesi, F, Kuprov, I, Rodgers, CT, Liddell, PA, Gust, D, Timmel, CR and Hore, PJ. (2008). Chemical compass model of avian magnetoreception. Nature, 453, 387-390.

Maeda, K, Robinson, AJ, Henbest, KB, Hogben, HJ, Biskup, T, Ahmad, M, Schleicher, E, Weber, S, Timmel, CR and Hore, PJ. (2012). Magnetically sensitive light-induced reactions in cryptochrome are consistent with its proposed role as a magnetoreceptor. Proceedings of the National Academy of Sciences, USA, 109, 4774-4779.

Manolopoulos, DE and Hore, PJ. (2013). An improved semiclassical theory of radical pair recombination reactions. Journal of Chemical Physics, 139, 124106.

Marais, A, Adams, B, Ringsmuth, AK, Ferretti, M, Gruber, JM, Hendrikx, R, Schuld, M, Smith, SL, Sinayskiy, I, Kruger, TPJ, Petruccione, F and Van Grondelle, R. (2018). The future of quantum biology. J. R. Soc. Interface, 15, 20180640.

Mohseni, M, Omar, Y, Engel, GS and Plenio, MB, eds. (2014). Quantum effects in biology. Cambridge: Cambridge University Press.

Mouritsen, H. (2018). Long-distance navigation and magnetoreception in migratory animals. Nature, 558, 50-59.

Mouritsen, H, Feenders, G, Liedvogel, M, Wada, K and Jarvis, ED. (2005). Night-vision brain area in migratory songbirds. Proceedings of the National Academy of Sciences, USA, 102, 8339-8344.

Mouritsen, H, Janssen-Bienhold, U, Liedvogel, M, Feenders, G, Stalleicken, J, Dirks, P and Weiler, R. (2004). Cryptochromes and neuronal-activity markers colocalize in the retina of migratory birds during magnetic orientation. Proceedings of the National Academy of Sciences, USA, 101, 14294-14299.

Müller, P and Ahmad, M. (2011). Light-activated cryptochrome reacts with molecular oxygen to form a flavin-superoxide radical pair consistent with magnetoreception. Journal of Biological Chemistry, 286, 21033-21040.

Nielsen, C, Kattnig, DR, Sjulstok, E, Hore, PJ and Solov'yov, IA. (2017). Ascorbic acid may not be involved in cryptochrome-based magnetoreception. J. R. Soc. Interface, 14, 20170657.

Nielsen, MA and Chuang, IL. (2010). Quantum computation and quantum information. New York: Cambridge University Press.

Niessner, C, Denzau, S, Gross, JC, Peichl, L, Bischof, HJ, Fleissner, G, Wiltschko, W and Wiltschko, R. (2011). Avian ultraviolet/violet cones identified as probable magnetoreceptors. Plos One, 6, e20091.

Niessner, C, Denzau, S, Peichl, L, Wiltschko, W and Wiltschko, R. (2014). Magnetoreception in birds: I. Immunohistochemical studies concerning the cryptochrome cycle. Journal of Experimental Biology, 217, 4221-4224. 
Niessner, C, Denzau, S, Stapput, K, Ahmad, M, Peichl, L, Wiltschko, W and Wiltschko, R. (2013). Magnetoreception: activated cryptochrome 1a concurs with magnetic orientation in birds. J. Roy. Soc. Interface, 10, 20130638.

Niessner, C, Gross, JC, Denzau, S, Peichl, L, Fleissner, G, Wiltschko, W and Wiltschko, R. (2016). Seasonally changing cryptochrome $1 \mathrm{~b}$ expression in the retinal ganglion cells of a migrating passerine bird. Plos One, 11, e 0150377.

Ritz, T, Adem, S and Schulten, K. (2000). A model for photoreceptor-based magnetoreception in birds. Biophysical Journal, 78, 707-718.

Ritz, T, Wiltschko, R, Hore, PJ, Rodgers, CT, Stapput, K, Thalau, P, Timmel, CR and Wiltschko, W. (2009). Magnetic compass of birds is based on a molecule with optimal directional sensitivity. Biophysical Journal, 96, 3451-3457.

Shannon, CE. (1948). A mathematical theory of communication. Bell System Tech. J., 27, 379-423.

Shannon, CE. (1959). Coding a discrete information source with a distortion measure. IRE Nat. Conv. Rec., 4, 142-163.

Sheppard, DMW, Li, J, Henbest, KB, Neil, SRT, Maeda, K, Storey, J, Schleicher, E, Biskup, T, Rodriguez, R, Weber, S, Hore, PJ, Timmel, CR and Mackenzie, SR. (2017). Millitesla magnetic field effects on the photocycle of Drosophila melanogaster cryptochrome. Sci. Rep., 7, 42228 .

Solov'yov, IA and Schulten, K. (2009). Magnetoreception through cryptochrome may involve superoxide. Biophysical Journal, 96, 4804-4813.

Steiner, UE and Ulrich, T. (1989). Magnetic field effects in chemical kinetics and related phenomena. Chemical Reviews, 89, 51-147.

Thomas, RJ, Szekely, T, Cuthill, IC, Harper, DGC, Newson, SE, Frayling, TD and Wallis, PD. (2002). Eye size in birds and the timing of song at dawn. Proc. R. Soc. B, 269, 831-837.

Timmel, CR, Till, U, Brocklehurst, B, Mclauchlan, KA and Hore, PJ. (1998). Effects of weak magnetic fields on free radical recombination reactions. Molec. Phys., 95, 71-89.

Vitalis, KM and Kominis, IK. (2017). Quantum-limited biochemical magnetometers designed using the Fisher information and quantum reaction control. Physical Review A, 95, 032129.

Voter, AF. (2007). Introduction to the kinetic Monte Carlo method. In Radiation effects in solids. NATO Science Series, vol. 235 eds. K. E. Sickafus, E. A. Kotomin and B. P. Uberuaga). Dordrecht: Springer.

Warrant, EJ. (1999). Seeing better at night: life style, eye design and the optimum strategy of spatial and temporal summation. Vision Research, 39, 1611-1630.

Wiltschko, R and Wiltschko, W. (1995). Magnetic orientation in animals. Berlin: Springer Verlag.

Wiltschko, W. (1968). Über den Einfluß statischer Magnetfelder auf die Zugorientierung der Rotkehlchen (Erithacus rubecula). Z. Tierpsychol., 25, 537-558. 
Wiltschko, W and Wiltschko, R. (1972). Magnetic compass of European robins. Science, 176, 6264.

Worster, S, Mouritsen, H and Hore, PJ. (2017). A light-dependent magnetoreception mechanism insensitive to light intensity and polarization. J. Roy. Soc. Interface, 14, 20170405.

Zapka, M, Heyers, D, Hein, CM, Engels, S, Schneider, NL, Hans, J, Weiler, S, Dreyer, D, Kishkinev, D, Wild, JM and Mouritsen, H. (2009). Visual but not trigeminal mediation of magnetic compass information in a migratory bird. Nature, 461, 1274-1278.

Zapka, M, Heyers, D, Liedvogel, M, Jarvis, ED and Mouritsen, H. (2010). Night-time neuronal activation of Cluster $\mathrm{N}$ in a day- and night-migrating songbird. European Journal of Neuroscience, 32, 619-624. 


\section{Figure captions}

Fig. 1. The radical pair mechanism. A model reaction scheme for a singlet-born radical pair able to react spin-selectively to form singlet and triplet reaction products with first order rate constants $k_{\mathrm{S}}$ and $k_{\mathrm{T}}$, respectively. The curly red-blue arrows represent the coherent interconversion of the two forms of the radical pair. The reaction scheme for radical pairs in cryptochrome is slightly more complicated (Hore and Mouritsen, 2016; Maeda et al., 2012).

Fig. 2. The time-dependence of the singlet probability of a model radical pair containing a ${ }^{1} \mathrm{H}$ nucleus in one of the radicals with hyperfine components $\left(A_{x x}, A_{y y}, A_{z z}\right)=(-0.5,-0.5,1.8) \mathrm{mT}$. $P_{\mathrm{S}}^{\alpha}\left(t_{\mathrm{R}}\right)$ is plotted for each of the two initial nuclear spin configurations. The applied magnetic field, $50 \mu \mathrm{T}$, is aligned with the symmetry axis $(z)$ of the hyperfine tensor. Spin relaxation, radical-radical interactions, and recombination reactions are not included.

Fig. 3. A schematic representation of the Information Theory approach. (a) The source of directional information is the geomagnetic field. $\theta$ is the bird's head direction with respect to geomagnetic north. (b) The head direction determines the magnetic field vector experienced by cryptochrome molecules in cells distributed across the retina. This information is encoded in the singlet reaction yield of light-induced radical pairs within the cryptochromes. (c) Each receptor cell $i$ experiences a different field direction and so has a different average singlet yield, $\Phi_{\mathrm{S}}^{i}(\Theta ; N)$, represented here as a visual modulation pattern (see later). (d) $\Phi_{\mathrm{S}}(\Theta ; N)$, the singlet yield signal from the whole of the retina, is decoded in the bird's eye and brain using some unknown data processing algorithm. (e) This results in an estimate of the head direction. The mutual information between different steps in the process is indicated.

Fig. 4. Calculation of the error in the estimate of the head direction for a model radical pair magnetic compass sensor. (a) Visual modulation patterns for different numbers, $N$, of photons per grouped receptor cell for a singlet yield anisotropy with $\sigma=26^{\circ}$ where each pixel represents $\Phi_{\mathrm{S}}^{i}(\Theta, N)$ for a single grouped receptor. (b) An example of the processing of $\Phi_{\mathrm{S}}(\Theta ; N)$ for $N$ $=10^{4}$. The orange trace is the diameter-averaged signal from the VMP plotted as a function of the azimuth. The blue trace is the result of fitting these data to a Gaussian model of the singlet yield anisotropy. (c) Comparison of the root-mean-squared error for the averaging and fitting methods with the Information Theory lower bound, $\epsilon_{\mathrm{LB}}$ as a function of the number of photons per grouped receptor cell. The inaccessible region where $\epsilon<\epsilon_{\mathrm{LB}}$ is shaded red. (d) VMPs in the $N \rightarrow \infty$ limit for different values of $\sigma$. (e) Lower bounds calculated for as a function of the width of the $\Phi_{\mathrm{S}}$ signal, $\sigma$. 
Fig. 5. The lower bound error in the head direction for the FAD-Trp radical pair state of cryptochrome as a function of the number of photons per grouped receptor cell. (a) A truncated 16-spin model with no exchange or dipolar coupling for lifetimes $\tau=1,2$ and $5 \mu$ s. (b) The full 29-spin model with $\tau=1 \mu$ s. The shaded regions indicate the expected number of photons per grouped receptor cell $\left(10^{2} \leq N \leq 10^{4}\right)$ when the light intensity is $\sim 3 \times 10^{-4}$ lux. 


\section{Table 1}

Lower bound head direction errors

\begin{tabular}{ccccc} 
radical pair $^{1}$ & nuclear spins $^{2}$ & $\tau / \mu \mathrm{s}$ & $\Delta \Phi_{\mathrm{S}}{ }^{3}$ & $\epsilon_{\mathrm{LB}}$ \\
\hline FAD-Trp & $7+7$ & 1 & $1.27 \times 10^{-3}$ & $26^{\circ}$ \\
FAD-Trp & $7+7$ & 5 & $3.69 \times 10^{-3}$ & $0.77^{\circ}$ \\
FAD-Trp & $15+12$ & 1 & $9.77 \times 10^{-5}$ & $86^{\circ}$ \\
FAD-Z & $7+0$ & 1 & $1.46 \times 10^{-1}$ & $\sim 0$ \\
FAD-Asc & $7+4$ & 1 & $6.66 \times 10^{-2}$ & $\sim 0$ \\
\hline
\end{tabular}

${ }^{1}$ Magnetic parameters given in Supplementary Information, Section IV. $N=1000$.

${ }^{2}$ Number of nuclear spins included in the FAD radical and the counter radical, respectively.

${ }^{3}$ Anisotropy of the singlet yield, defined as $\Delta \Phi_{\mathrm{S}}=\max \left[\Phi_{\mathrm{S}}\right]-\min \left[\Phi_{\mathrm{S}}\right]$. 


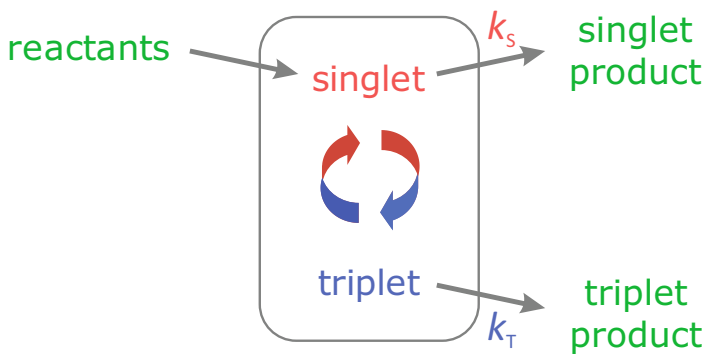

radical pair 


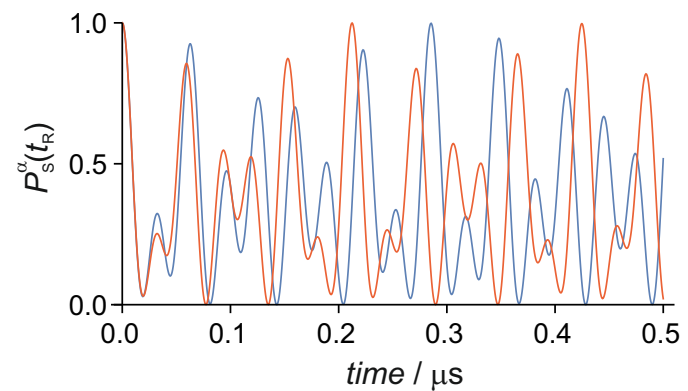




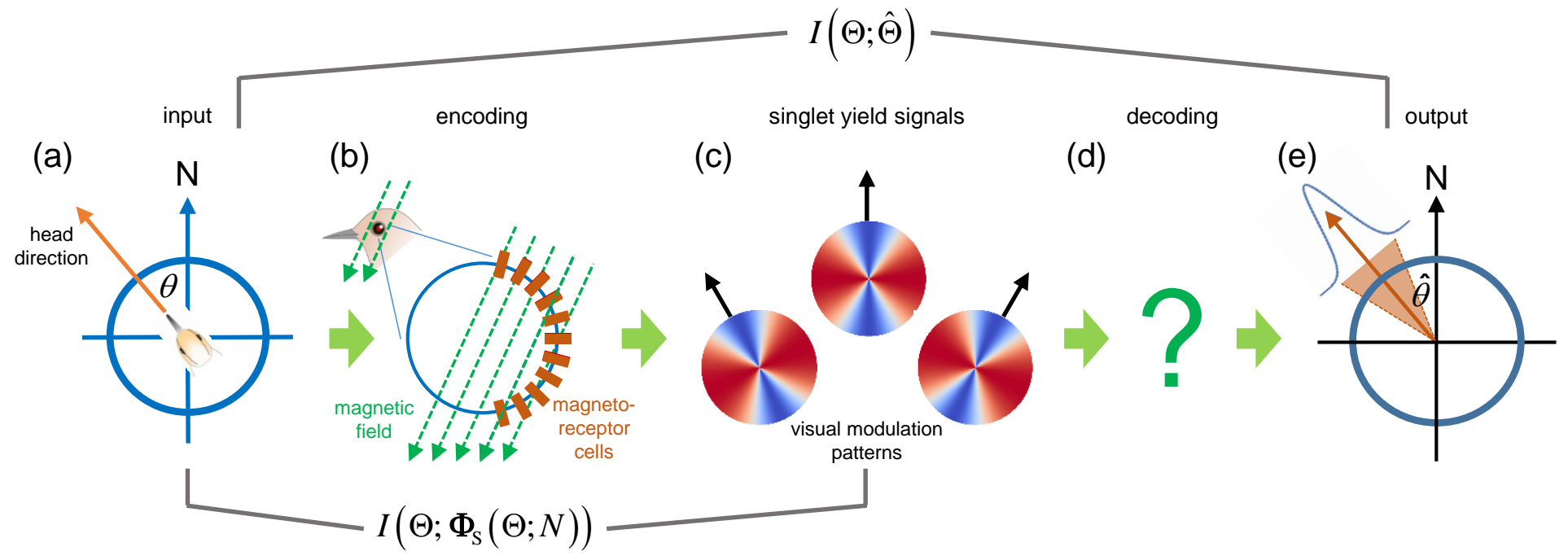


(a)
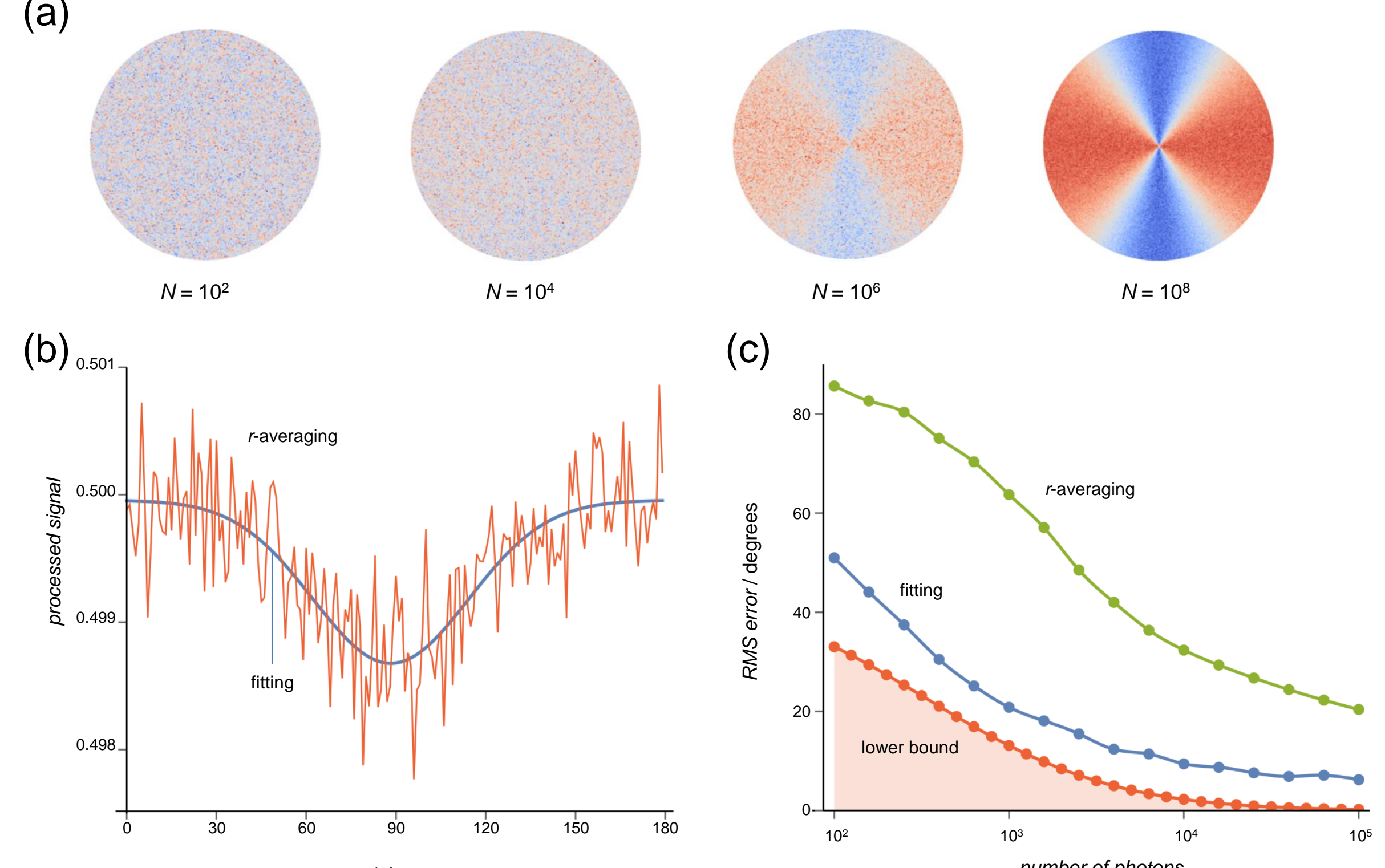

(d)

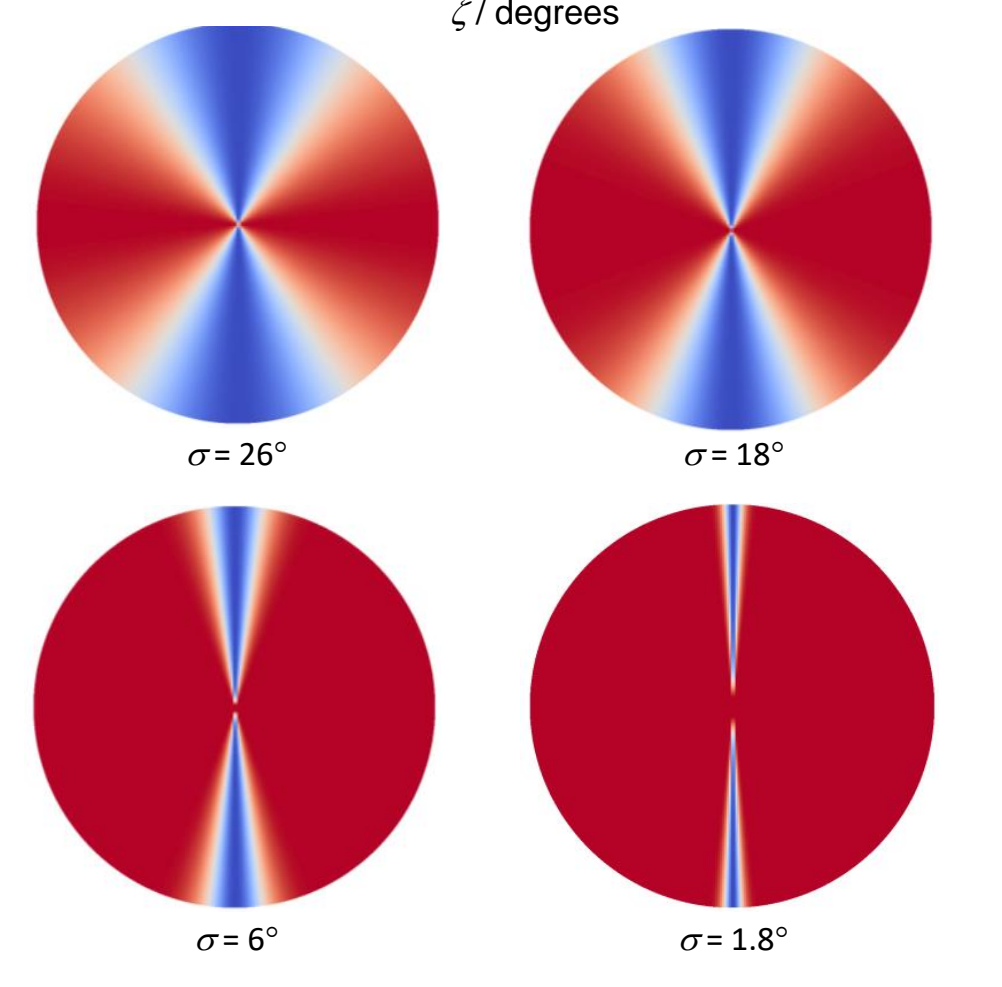

(e)

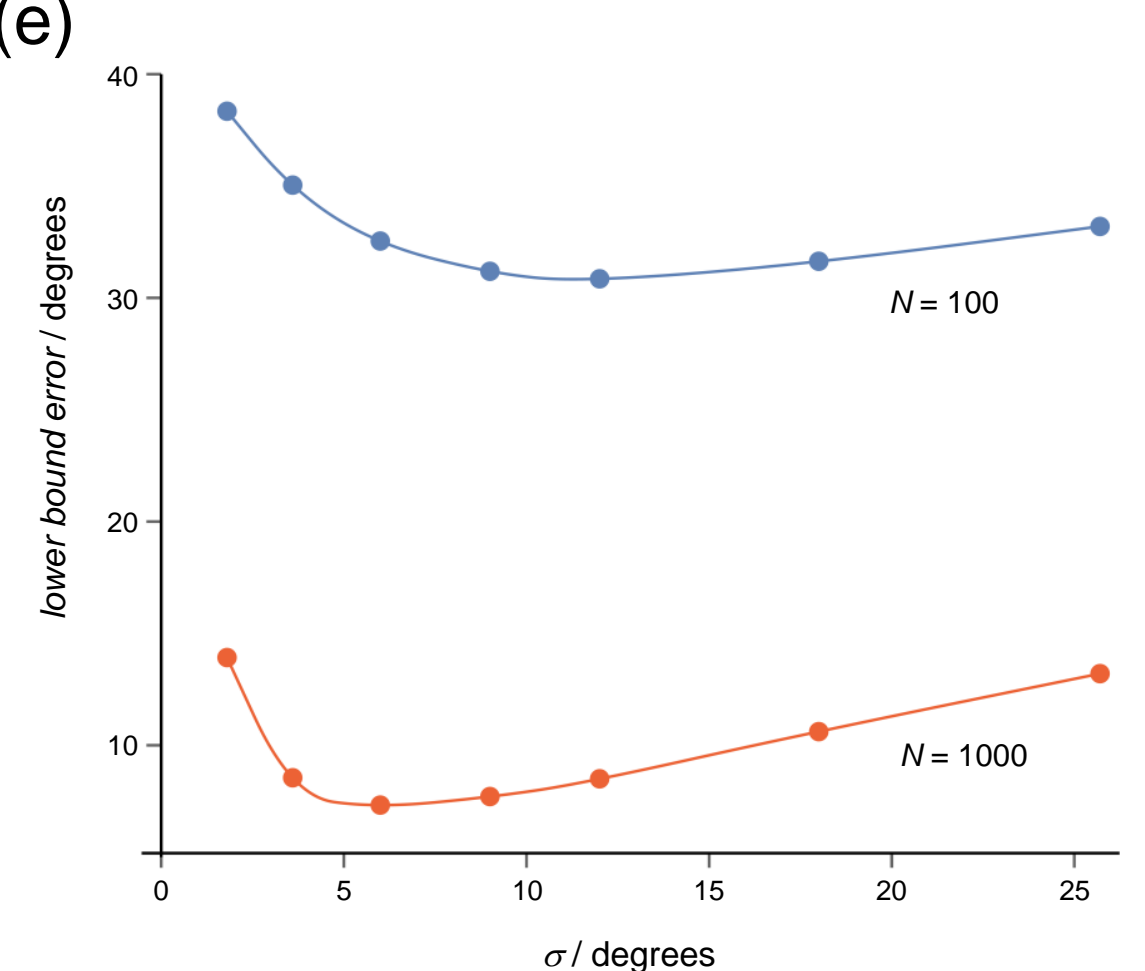



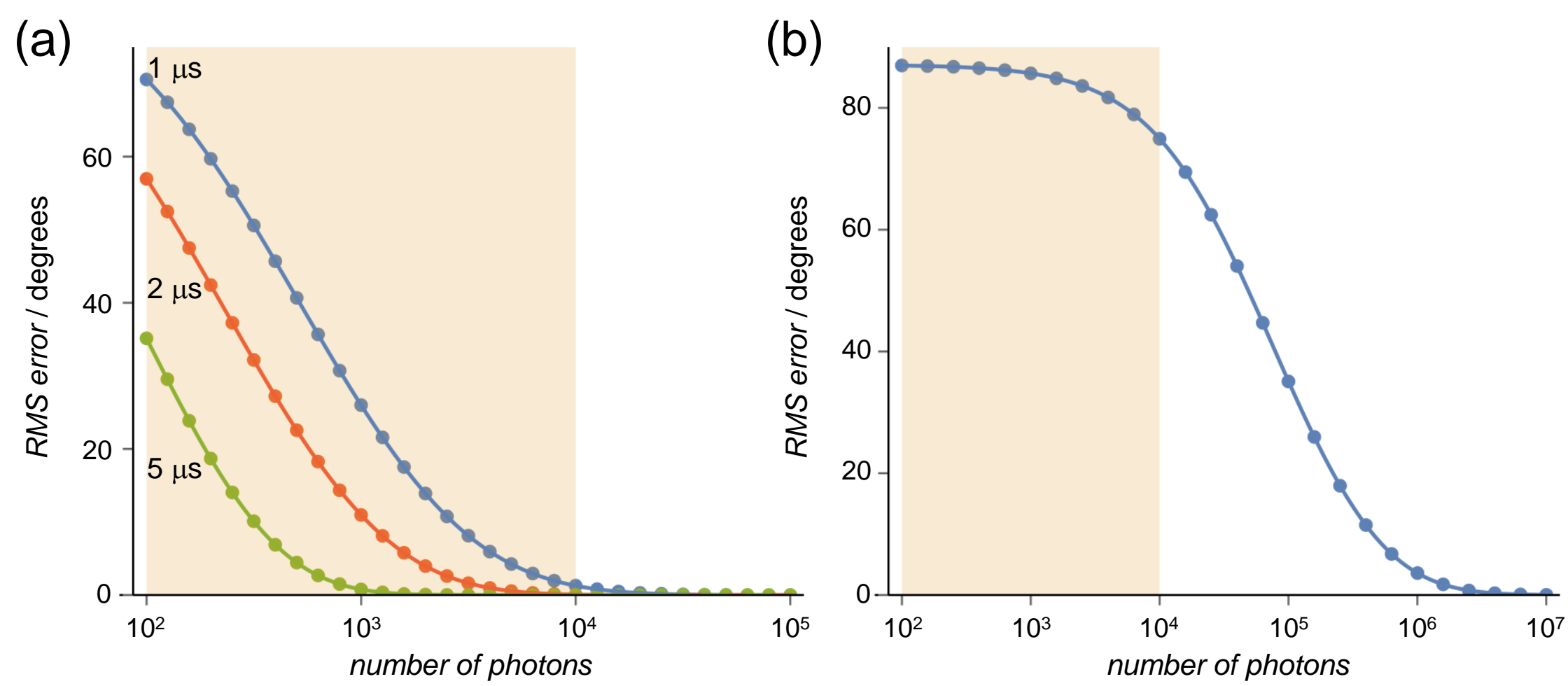


\title{
Navigating at night: fundamental limits on the sensitivity of radical pair magnetoreception under dim light
}

\author{
H. G. Hiscock, T. W. Hiscock, D. R. Kattnig, T. Scrivener,
}

A. M. Lewis, D. E. Manolopoulos, and P. J. Hore

\section{S. I Distribution of reaction yields}

We derived the distribution of the singlet yield $\left(\Phi_{\mathrm{S}}(N)\right)$ with a finite number $(N)$ of reaction cycles given a known average singlet yield $\left(\Phi_{\mathrm{S}}\right)$ (Equation $\left.(3)\right)$ :

$$
N \Phi_{\mathrm{S}}(N) \sim \mathcal{B}\left(N, \Phi_{\mathrm{S}}\right)
$$

We can numerically verify this result using a Kinetic Monte Carlo (KMC) algorithm [1]. We calculate the singlet yield $\Phi_{\mathrm{S}}(N)$ for $N$ reactions by explicitly computing the outcome of $N$ reaction trajectories, and then compare the distribution of many calculated singlet yields against the expected binomial distribution in Equation (S.1). With $k_{\mathrm{S}}=k_{\mathrm{T}}=k$ (Figure 1), the calculation has the following steps:

1. Randomly select an initial nuclear spin configuration and combine it with a pure singlet electron spin state: $\left|\Psi^{\alpha}(0)\right\rangle$.

2. Propagate this initial state to $t_{\mathrm{R}}=k^{-1} \ln \left(1 / u_{1}\right)$ where $u_{1} \in[0,1)$ is a random number between 0 and 1: $\left|\Psi^{\alpha}\left(t_{\mathrm{R}}\right)\right\rangle=\exp \left(-i \hat{H} t_{\mathrm{R}}\right)\left|\Psi^{\alpha}(0)\right\rangle$, where $\hat{H}$ is the spin Hamiltonian of the radical pair.

3. Choose a second random number, $u_{2} \in[0,1)$. If $u_{2}<P_{\mathrm{S}}^{\alpha}\left(t_{\mathrm{R}}\right)$ then the trajectory forms the singlet product, otherwise the triplet product is formed. $P_{\mathrm{S}}^{\alpha}\left(t_{\mathrm{R}}\right)=\left\langle\Psi^{\alpha}\left(t_{\mathrm{R}}\right)\left|\hat{P}_{\mathrm{S}}\right| \Psi^{\alpha}\left(t_{\mathrm{R}}\right)\right\rangle$ 
where $\hat{P}_{\mathrm{S}}$ the singlet projection operator.

4. Repeat this procedure $N$ times and compute the fractional singlet yield, $\Phi_{\mathrm{S}}(N)$.

We use this KMC procedure to compute $\Phi_{\mathrm{S}}\left(10^{5}\right)$, i.e. the fractional singlet yield averaged over $N=10^{5}$ reactions, for a model 3 -spin radical pair containing a single ${ }^{1} \mathrm{H}$ nucleus with hyperfine components $\left(A_{x x}, A_{y y}, A_{z z}\right)=(-0.0989,-0.0989,1.752) \mathrm{mT}$. The calculation is repeated $10^{5}$ times to approximate the distribution of $\Phi_{\mathrm{S}}(N)$.

The computed data is compared with the expected binomial distribution in Equation (S.1) using a quantile-quantile (Q-Q) plot as shown in Figure S.1. A Q-Q plot shows the positions of the quantiles (the $i^{\text {th }}$ quantile is the value below which $i \%$ of the data falls) of one set of data against another; the two distributions are equivalent if the points fall on the line with unit gradient passing through the origin [2]. Clearly this is the case in Figure S.1, verifying our derivation of Equation (S.1).

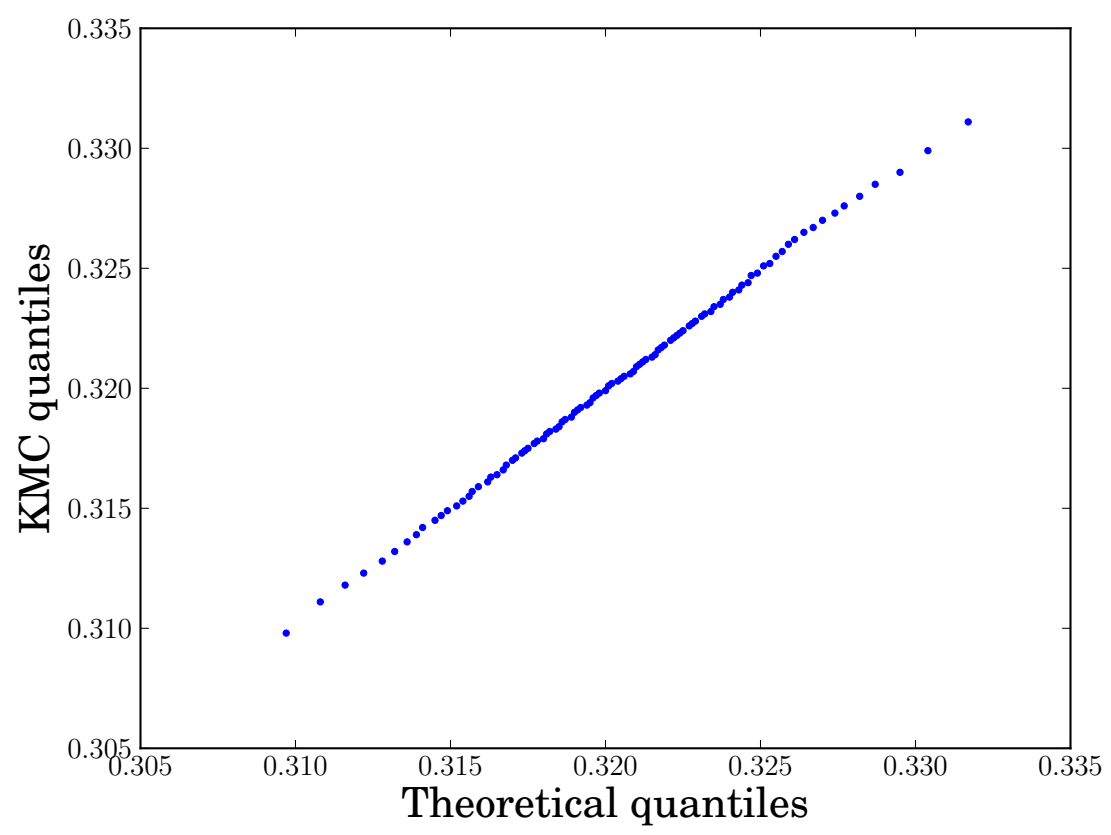

Figure S.1: A quantile-quantile plot comparing the distribution of singlet yields calculated using the KMC algorithm with the quantiles expected from Equation (S.1). The results shown are for $N=10^{5}$ and $10^{5} \mathrm{KMC}$ calculations. 


\section{S. II Computing the lower bound error in the head direction}

Our expression for the lower bound of the root-mean-squared (RMS) error in the head direction estimate given in Equation (15) is:

$$
\epsilon^{2} \geq \epsilon_{\mathrm{LB}}^{2}=\frac{2 \pi}{e} \exp \left[-2 I\left(\Theta ; \boldsymbol{\Phi}_{\mathrm{S}}(\Theta ; N)\right)\right]
$$

In order to compute the error bound, we need to calculate the mutual information between the head direction, $\Theta$, and the singlet yield signal, $\mathbf{\Phi}_{\mathrm{S}}(\Theta ; N)$. This involves calculating the two entropy terms that make up the mutual information, which we choose to write $(I(X ; Y)$ being symmetric)

$$
I\left(\Theta ; \boldsymbol{\Phi}_{\mathrm{S}}(\Theta ; N)\right) \equiv I\left(\boldsymbol{\Phi}_{\mathrm{S}}(\Theta ; N) ; \Theta\right)=h\left(\boldsymbol{\Phi}_{\mathrm{S}}(\Theta ; N)\right)-h\left(\boldsymbol{\Phi}_{\mathrm{S}}(\Theta ; N) \mid \Theta\right) .
$$

In the following, we will use the Gaussian approximation of the singlet yield probability distribution for large $N$ (from the central limit theorem, Equation (3))

$$
\Phi_{\mathrm{S}}(N) \sim \mathcal{N}\left(\Phi_{\mathrm{S}}, \Phi_{\mathrm{S}}\left(1-\Phi_{\mathrm{S}}\right) / N\right)
$$

For a given head direction $\Theta=\theta$ (we use upper case to denote random variables and lower case to denote a specific value of the corresponding variable), the mean and variance at each of the $N_{\text {rec }}$ grouped receptor cells (labelled by $i$ ) can be written in terms of the average singlet yield $\Phi_{\mathrm{S}}^{i}(\theta)$ :

$$
\begin{aligned}
& \mu_{i \mid \theta}=\Phi_{\mathrm{S}}^{i}(\theta) \\
& \sigma_{i \mid \theta}^{2}=\Phi_{\mathrm{S}}^{i}(\theta)\left(1-\Phi_{\mathrm{S}}^{i}(\theta)\right) / N
\end{aligned}
$$

so that the probability density function of the singlet yield signal $p\left(\boldsymbol{\Phi}_{\mathrm{S}}(\Theta ; N)=\phi_{\mathrm{S}}(N) \mid \Theta=\theta\right)$ $=p\left(\phi_{\mathrm{S}}(N) \mid \theta\right)$ given a head direction $\theta$ and number of photons $N$ is

$$
p\left(\phi_{\mathrm{S}}(N) \mid \theta\right)=\prod_{i=1}^{N_{\text {receptors }}} \mathcal{N}\left(\mu_{i \mid \theta}, \sigma_{i \mid \theta}^{2}\right)
$$


The other important result that will be used below is the law of total probability [3], which for our purposes takes the form

$$
\begin{aligned}
p\left(\phi_{\mathrm{S}}(N)\right) & =\int p\left(\boldsymbol{\phi}_{\mathrm{S}}(N) \mid \theta\right) p(\theta) \mathrm{d} \theta \\
& =\sum_{k=1}^{K} p\left(\boldsymbol{\phi}_{\mathrm{S}}(N) \mid \theta_{k}\right) P\left(\theta_{k}\right) .
\end{aligned}
$$

In other words, we can describe the probability density of $\boldsymbol{\Phi}_{\mathrm{S}}(\Theta ; N)$ as the distribution of the singlet yield signal for a fixed $\theta$ averaged over all values of $\theta$. In the second line, we have replaced the integral over $\theta$ with a sum over discrete values of $\theta_{k}$, as we will evaluate the integral using numerical quadrature, not having a closed form expression for how the average singlet yield signal varies with $\theta \cdot P\left(\theta_{k}\right)=1 / K$ where $K$ is the number of values of $\theta$ sampled to calculate the integral. We use $K=1000$ to converge the directional RMS error to within $1^{\circ}$

\section{S. II. A. The conditional entropy}

Calculating the conditional entropy is relatively straightforward as, for a given value of $\theta$, the singlet yield at each receptor is described by an independent Gaussian

$$
\begin{aligned}
h\left(\boldsymbol{\Phi}_{\mathrm{S}}(\Theta ; N) \mid \Theta\right) & =-\iint p\left(\boldsymbol{\phi}_{\mathrm{S}}(N), \theta\right) \ln \left(p\left(\boldsymbol{\phi}_{\mathrm{S}}(N) \mid \theta\right)\right) \mathrm{d} \boldsymbol{\phi}_{\mathrm{S}}(N) \mathrm{d} \theta \\
& \approx-\frac{1}{K} \sum_{k=1}^{K} \int p\left(\phi_{\mathrm{S}}(N) \mid \theta_{k}\right) \ln \left(p\left(\phi_{\mathrm{S}}(N) \mid \theta_{k}\right)\right) \mathrm{d} \boldsymbol{\phi}_{\mathrm{S}}(N),
\end{aligned}
$$

where we have used $p\left(\phi_{\mathrm{S}}(N), \theta\right)=p\left(\phi_{\mathrm{S}}(N) \mid \theta\right) P(\theta)$ in going from the first line to the second. As stated above, we are going to numerically sample over the distribution of $\Theta$, and the integral over $\theta$ is approximated by a summation.

For each value of $\theta_{k}$, the integral exactly corresponds to the entropy of a multivariate Gaussian, whose entropy is given by the multivariate generalisation of Equation (7)

$$
h(\mathbf{X} \sim \mathcal{N}(\boldsymbol{\mu}, \Sigma))=\frac{1}{2} \ln \left((2 \pi e)^{n} \operatorname{det}(\Sigma)\right)
$$


where $\Sigma$ is the $n \times n$ covariance matrix of the distribution, with elements

$$
\Sigma_{i j}=\operatorname{cov}\left[X_{i}, X_{j}\right]=E\left[\left(X_{i}-E\left[X_{i}\right]\right)\left(X_{j}-E\left[X_{j}\right]\right)\right]
$$

Since the singlet yield at each receptor is independent for a fixed $\theta$, the covariance matrix of this distribution is diagonal, meaning the total entropy is the sum of entropies of independent

Gaussian variables representing the singlet yield at each receptor. We can therefore write the conditional entropy as:

$$
h\left(\mathbf{\Phi}_{\mathrm{S}}(\Theta ; N) \mid \Theta\right)=\frac{1}{2 K} \sum_{k=1}^{K} \sum_{i=1}^{N_{\mathrm{rec}}} \ln \left(2 \pi e \sigma_{i \mid \theta_{k}}^{2}\right) .
$$

\section{S. II. B. Signal entropy}

The other quantity left to calculate is the entropy of the singlet yield signal, $h\left(\boldsymbol{\Phi}_{\mathrm{S}}(\Theta ; N)\right)$. Because of the variation of $\Phi_{\mathrm{S}}$ with $\theta$, the singlet yield at each receptor is not independent. For a pair of receptors, their singlet yield signals are correlated because of the fixed geometrical relation between them. Additionally, the distribution of $\Phi_{\mathrm{S}}^{i}(\Theta ; N)$ is not simply described by a Gaussian. Therefore we cannot straightforwardly evaluate the entropy. Instead, we compute the upper bound on the entropy using the multivariate Gaussian limit $[4] h(\mathbf{X}) \leq$ $h(\mathbf{X} \sim \mathcal{N}(\boldsymbol{\mu}, \Sigma))$ (which is compatible with the bound in Equation (S.2)).

In order to compute this upper bound, we need to evaluate the elements of the covariance matrix, and so consider the joint probability distribution of pairs of receptors. We again use the law of total probability (Equation (S.7)) and the fact that the singlet yield at different receptors is independent for a given $\theta$

$$
\begin{aligned}
p\left(\phi_{\mathrm{S}}^{i}(N), \phi_{\mathrm{S}}^{j}(N)\right) & =\sum_{k=1}^{K} p\left(\phi_{\mathrm{S}}^{i}(N), \phi_{\mathrm{S}}^{j}(N) \mid \theta_{k}\right) P\left(\theta_{k}\right) \\
& =\frac{1}{K} \sum_{k=1}^{K} p\left(\phi_{\mathrm{S}}^{i}(N) \mid \theta_{k}\right) p\left(\phi_{\mathrm{S}}^{j}(N) \mid \theta_{k}\right)
\end{aligned}
$$


We can use this to evaluate the $i, j$ element in the covariance matrix (where $\bar{\Phi}_{\mathrm{S}}^{i}$ is the average singlet yield at receptor $i$ over all $\theta$ )

$$
\begin{aligned}
E & {\left[\left(\phi_{\mathrm{S}}^{i}(N)-\bar{\Phi}_{\mathrm{S}}^{i}\right)\left(\phi_{\mathrm{S}}^{j}(N)-\bar{\Phi}_{\mathrm{S}}^{j}\right)\right] } \\
& \left.=\iint p\left(\phi_{\mathrm{S}}^{i}(N), \phi_{\mathrm{S}}^{j}(N)\right)\right)\left(\phi_{\mathrm{S}}^{i}(N)-\bar{\Phi}_{\mathrm{S}}^{i}\right)\left(\phi_{\mathrm{S}}^{j}(N)-\bar{\Phi}_{\mathrm{S}}^{j}\right) \mathrm{d} \phi_{\mathrm{S}}^{i}(N) \mathrm{d} \phi_{\mathrm{S}}^{j}(N) \\
& =\frac{1}{K} \sum_{k=1}^{K} \iint p\left(\phi_{\mathrm{S}}^{i}(N) \mid \theta_{k}\right) p\left(\phi_{\mathrm{S}}^{j}(N) \mid \theta_{k}\right)\left(\phi_{\mathrm{S}}^{i}(N)-\bar{\Phi}_{\mathrm{S}}^{i}\right)\left(\phi_{\mathrm{S}}^{j}(N)-\bar{\Phi}_{\mathrm{S}}^{j}\right) \mathrm{d} \phi_{\mathrm{S}}^{i}(N) \mathrm{d} \phi_{\mathrm{S}}^{j}(N) \\
& =\frac{1}{K} \sum_{k=1}^{K} E\left[\Phi_{\mathrm{S}}^{i}\left(\theta_{k}, N\right)-\bar{\Phi}_{\mathrm{S}}^{i}\right] \times E\left[\Phi_{\mathrm{S}}^{j}\left(\theta_{k}, N\right)-\bar{\Phi}_{\mathrm{S}}^{j}\right]
\end{aligned}
$$

meaning the off-diagonal elements in the covariance matrix are simply the average of the covariance over all values of $\theta_{k}$, which can be easily computed

$$
\begin{aligned}
\operatorname{cov}[ & {\left[\Phi_{\mathrm{S}}^{i}\left(\theta_{k}, N\right), \Phi_{\mathrm{S}}^{j}\left(\theta_{k}, N\right)\right]_{\theta_{k}} } \\
& =E\left[\Phi_{\mathrm{S}}^{i}\left(\theta_{k}, N\right)-\bar{\Phi}_{\mathrm{S}}^{i}\right] \times E\left[\Phi_{\mathrm{S}}^{j}\left(\theta_{k}, N\right)-\bar{\Phi}_{\mathrm{S}}^{j}\right] \\
& =\left(\mu_{i \mid \theta_{k}}-\bar{\Phi}_{\mathrm{S}}^{i}\right)\left(\mu_{j \mid \theta_{k}}-\bar{\Phi}_{\mathrm{S}}^{j}\right) .
\end{aligned}
$$

Similarly, the diagonal components can be computed at each $\theta_{k}$

$$
\begin{aligned}
\operatorname{cov} & {\left[\Phi_{\mathrm{S}}^{i}\left(\theta_{k}, N\right), \Phi_{\mathrm{S}}^{i}\left(\theta_{k}, N\right)\right]_{\theta_{k}} } \\
& =E\left[\left(\Phi_{\mathrm{S}}^{i}\left(\theta_{k}, N\right)-\bar{\Phi}_{\mathrm{S}}^{i}\right)^{2}\right] \\
& =\sigma_{i \mid \theta_{k}}^{2}+\mu_{i \mid \theta_{k}}^{2}-2 \mu_{i \mid \theta_{k}} \bar{\Phi}_{\mathrm{S}}^{i}+\left(\bar{\Phi}_{\mathrm{S}}^{i}\right)^{2} .
\end{aligned}
$$

Using these expressions, we can finally compute the total covariance matrix

$$
\left(\Sigma_{\boldsymbol{\Phi}_{\mathrm{S}}(\Theta ; N)}\right)_{i j}=\frac{1}{K} \sum_{k=1}^{K} \operatorname{cov}\left[\Phi_{\mathrm{S}}^{i}\left(\theta_{k}, N\right), \Phi_{\mathrm{S}}^{j}\left(\theta_{k}, N\right)\right]_{\theta_{k}}
$$

meaning the entropy is bounded by

$$
h\left(\boldsymbol{\Phi}_{\mathrm{S}}(\Theta ; N)\right) \leq \frac{1}{2} \ln \left((2 \pi e)^{N_{\mathrm{rec}}} \operatorname{det}\left(\Sigma_{\boldsymbol{\Phi}_{\mathrm{S}}(\Theta ; N)}\right) .\right.
$$




\section{S. II. C. Performing the calculation}

Combining the results from the previous sections (Equation (S.11) and Equation (S.17)), we can write the mutual information as

$$
\begin{aligned}
I\left(\Theta ; \boldsymbol{\Phi}_{\mathrm{S}}(\Theta ; N)\right) & =h\left(\boldsymbol{\Phi}_{\mathrm{S}}(\Theta ; N)\right)-h\left(\boldsymbol{\Phi}_{\mathrm{S}}(\Theta ; N) \mid \Theta\right) \\
& \leq \frac{1}{2} \ln \left((2 \pi e)^{N_{\mathrm{rec}}} \operatorname{det}\left(\Sigma_{\boldsymbol{\Phi}_{\mathrm{S}}(\Theta ; N)}\right)\right)-\frac{1}{2 K} \sum_{k=1}^{K} \sum_{i=1}^{N_{\mathrm{rec}}} \ln \left(2 \pi e \sigma_{i \mid \theta_{k}}^{2}\right) .
\end{aligned}
$$

Finally, we insert this into Equation (S.2) to find an expression for the strict lower bound on the error in the estimator $(\hat{\Theta})$ of the head direction $(\Theta)$ based on the singlet yield signal $\left(\boldsymbol{\Phi}_{\mathrm{S}}(\Theta ; N)\right)$ with $N$ reactions cycles per grouped magnetoreceptor cell

$$
\begin{gathered}
\epsilon^{2} \geq \frac{1}{2 \pi e} \exp \left(2 \ln (2 \pi)-\ln \left((2 \pi e)^{N_{\text {rec }}} \operatorname{det}\left(\Sigma_{\boldsymbol{\Phi}_{\mathrm{S}}(\Theta ; N)}\right)\right)\right. \\
\left.+\frac{1}{K} \sum_{k=1}^{K} \sum_{i=1}^{N_{\text {rec }}} \ln \left(2 \pi e \sigma_{i \mid \theta_{k}}^{2}\right)\right) \\
=\frac{2 \pi}{e} \times \frac{1}{(2 \pi e)^{N_{\text {rec }}} \operatorname{det}\left(\Sigma_{\boldsymbol{\Phi}_{\mathrm{S}}(\Theta ; N)}\right)} \times \prod_{k=1}^{K} \prod_{i=1}^{N_{\text {rec }}}\left(2 \pi e \sigma_{i \mid \theta_{k}}^{2}\right)^{1 / K} .
\end{gathered}
$$




\section{S. III The magnetic field direction in the radical pair frame}

Here we outline the geometric manipulations required to transform the magnetic field vector from the laboratory frame into the radical pair (RP) frame.

We assume for simplicity that the eye of a bird is spherical. As in the main text, we denote the head direction by $\theta$ and define $\theta=0^{\circ}$ to mean that the bird is looking directly towards magnetic North. The other angle parameterising the field direction is the inclination angle $(\phi)$ which is taken as $66^{\circ}$ (we assume that the bird is always looking horizontally). Together these angles give the external field vector in the retina frame (the $z$-axis is defined as the symmetry axis of the eye) [5]

$$
\mathbf{B}_{\mathrm{R}}=(\sin (\chi) \cos (\psi), \sin (\chi) \sin (\psi), \cos (\chi)),
$$

where

$$
\begin{aligned}
& \chi=\arccos (-\cos (\theta) \cos (\phi)), \\
& \psi=\arctan (-\cos (\phi) \sin (\theta) / \sin (\phi)) .
\end{aligned}
$$

In order to calculate the singlet yield, $\Phi_{\mathrm{S}}$, at each receptor, we require the field direction in the RP frame. First we must transform to the magnetoreceptor cell frame before accounting for the relative orientation of the RP within the receptor cell [5]. For simplicity, it is assumed that all RPs within a single receptor cell are perfectly aligned and that all receptor cells are identical. The receptor cell orientation is defined by the angles $\{\kappa, \nu, \eta\}$. The cell is presumed to be normal to the retina surface, and the first two angles are determined by the position of

the cell on the retina. If $x$ and $y$ are the coordinates (normalised such that $x^{2}+y^{2} \leq 1$ ) of the cell when the retina is projected onto a plane [5] (using an equal angle projection)

$$
\begin{aligned}
& \kappa=2 \arctan \left(\sqrt{x^{2}+y^{2}}\right), \\
& \nu=\arctan (x / y) .
\end{aligned}
$$


The angle $\eta$ gives the rotation about the long $(z)$ axis of the cell. The choice of this angle is arbitrary as it combines with the $z$ orientation of the RP within the cell [6]. The orientation of the RP within the cell is defined by angles $\{\alpha, \beta, \gamma\}$.

With these angles in hand, we can define the rotation which rotates the field vector in the retina frame to the RP frame [5] (see Figure 3 in [6] for a diagrammatic representation of this geometric relation)

$$
\mathrm{R}=\mathrm{R}_{z}(\nu) \mathrm{R}_{y}(\kappa) \mathrm{R}_{z}(\eta) \mathrm{R}_{z}(\alpha) \mathrm{R}_{y}(\beta) \mathrm{R}_{z}(\gamma)
$$

where

$$
\mathrm{R}_{y}(\lambda)=\left(\begin{array}{ccc}
\cos (\lambda) & 0 & \sin (\lambda) \\
0 & 1 & 0 \\
-\sin (\lambda) & 0 & \cos (\lambda)
\end{array}\right), \mathrm{R}_{z}(\lambda)=\left(\begin{array}{ccc}
\cos (\lambda) & -\sin (\lambda) & 0 \\
\sin (\lambda) & \cos (\lambda) & 0 \\
0 & 0 & 1
\end{array}\right)
$$

Using this rotation matrix, we can find the field vector in the RP frame

$$
\begin{aligned}
\mathbf{B}_{\mathrm{RP}} & =\mathrm{R}^{\mathrm{T}} \mathbf{B}_{\mathrm{R}} \\
& =(\sin (\zeta) \cos (\delta), \sin (\zeta) \sin (\delta), \cos (\zeta))
\end{aligned}
$$

and so finally we have the (average) singlet yield at the receptor $i$ whose position in the retina is defined by $x$ and $y$ with the bird's head direction $(\theta)$ (and fixed inclination of the external field) [5]

$$
\Phi_{\mathrm{S}}^{i}(\theta) \equiv \Phi_{\mathrm{S}}(\zeta, \delta)
$$

In order to perform the calculations presented in the main text, it is necessary to make some arbitrary choices about values of the unknown angles above. We choose $\alpha+\eta=\beta=$ $\pi / 2$, and $\gamma=0$. We assume that the exact choice of these angles is not important for our analysis [7], but it should be noted that this does assume perfect orientation of the RPs in three dimensions in all the receptor cells. This choice of angles means that, when $\theta=0$, $\cos (\zeta)=\sin (\nu) \sin (\phi)$ (Equation (16)) 


\section{S. IV Hyperfine tensors}

\section{S. IV. A. FAD Radical}

The hyperfine tensors of the FAD anion radical were calculated using density functional theory (DFT) in Gaussian-03 [8] at the UB3LYP/EPR-III level [9]. The H8 and H7 methyl groups are assumed to rotate quickly enough to average the hyperfine tensors of the three protons, and their anisotropic components are very small and are therefore neglected. The $\mathrm{H} \beta$ protons were assigned isotropic hyperfine couplings equal to the largest of the components for the methyl group in lumiflavin [10].

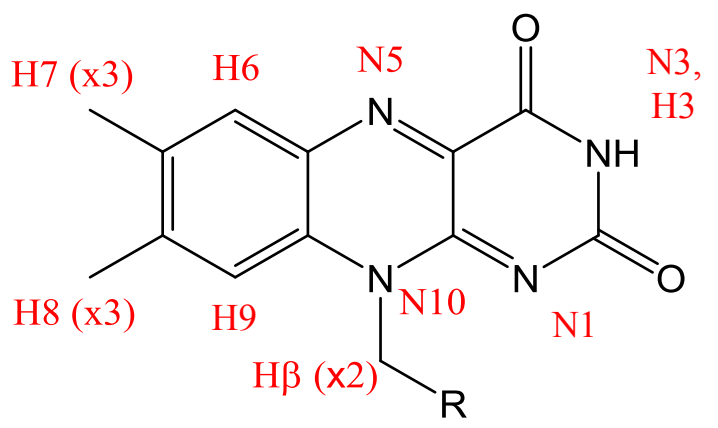

Figure S.2: Nucleus labels of $\mathrm{FAD}^{\bullet-}$ radical in DFT calculations.

\begin{tabular}{|c|c|c|c|c|c|}
\hline Nucleus & & $\mathbf{A} / \mathrm{mT}$ & & $a_{\text {iso }} / \mathrm{mT}$ & $T_{q q} / \mathrm{mT}$ \\
\hline \multirow{3}{*}{ N5 } & -0.0989 & 0.0039 & 0.0 & \multirow{3}{*}{0.5233} & 1.2336 \\
\hline & 0.0039 & -0.0881 & 0.0 & & -0.6101 \\
\hline & 0.0 & 0.0 & 1.7569 & & -0.6234 \\
\hline \multirow{3}{*}{ N10 } & -0.0190 & -0.0048 & 0.0 & \multirow{3}{*}{0.1887} & 0.4159 \\
\hline & -0.0048 & -0.0196 & 0.0 & & -0.2031 \\
\hline & 0.0 & 0.0 & 0.6046 & & -0.2128 \\
\hline \multirow{3}{*}{ H6 } & -0.2569 & -0.1273 & 0.0 & \multirow{3}{*}{-0.3872} & 0.1896 \\
\hline & -0.1273 & -0.4711 & 0.0 & & -0.0464 \\
\hline & 0.0 & 0.0 & -0.4336 & & -0.1432 \\
\hline
\end{tabular}




\begin{tabular}{|c|c|c|c|c|c|}
\hline \multirow{3}{*}{$\mathrm{H} 8(\times 3)$} & 0.4399 & 0.0 & 0.0 & \multirow{3}{*}{0.4399} & 0.0 \\
\hline & 0.0 & 0.4399 & 0.0 & & 0.0 \\
\hline & 0.0 & 0.0 & 0.4399 & & 0.0 \\
\hline \multirow{3}{*}{$\mathrm{H} \beta(\times 2)$} & 0.4070 & 0.0 & 0.0 & \multirow{3}{*}{0.4070} & 0.0 \\
\hline & 0.0 & 0.4070 & 0.0 & & 0.0 \\
\hline & 0.0 & 0.0 & 0.4070 & & 0.0 \\
\hline \multirow{3}{*}{$\mathrm{H} 7(\times 3)$} & -0.1416 & 0.0 & 0.0 & \multirow{3}{*}{-0.1416} & 0.0 \\
\hline & 0.0 & -0.1416 & 0.0 & & 0.0 \\
\hline & 0.0 & 0.0 & -0.1416 & & 0.0 \\
\hline \multirow{3}{*}{ H9 } & 0.0554 & 0.0057 & 0.0 & \multirow{3}{*}{0.0565} & -0.0016 \\
\hline & 0.0057 & 0.1191 & 0.0 & & 0.0631 \\
\hline & 0.0 & 0.0 & -0.0050 & & -0.0615 \\
\hline \multirow{3}{*}{ H3 } & -0.0146 & 0.0286 & 0.0 & \multirow{3}{*}{-0.0175} & -0.01171 \\
\hline & 0.0286 & 0.0226 & 0.0 & & 0.0588 \\
\hline & 0.0 & 0.0 & -0.0646 & & -0.0471 \\
\hline \multirow{3}{*}{ N3 } & -0.0404 & 0.0045 & 0.0 & \multirow{3}{*}{-0.0383} & -0.0048 \\
\hline & 0.0045 & -0.0358 & 0.0 & & 0.0053 \\
\hline & 0.0 & 0.0 & -0.0388 & & -0.0005 \\
\hline \multirow{3}{*}{ N1 } & -0.0225 & 0.0025 & 0.0 & \multirow{3}{*}{-0.0035} & -0.0177 \\
\hline & 0.0025 & -0.0259 & 0.0 & & -0.0238 \\
\hline & 0.0 & 0.0 & 0.0380 & & 0.0415 \\
\hline
\end{tabular}

Table 1: Hyperfine tensors $(\mathbf{A})$ for the $\mathrm{FAD}^{\bullet-}$ radical [10].

The isotropic $\left(a_{\text {iso }} / \mathrm{mT}\right)$ and anisotropic $\left(T_{q q} / \mathrm{mT}\right)$ interactions are given. 


\section{S. IV. B. Tryptophan Radical}

The hyperfine tensors of the tryptophan cation radical were calculated using DFT in Gaussian03 [8] at the UB3LYP/EPR-III level [9]. These tensors are in the same axis system as the FAD radical as above, appropriate for the relative orientation of the FAD cofactor and Trp-342 in the crystal structure of $D m \mathrm{CRY}[11,12]$.

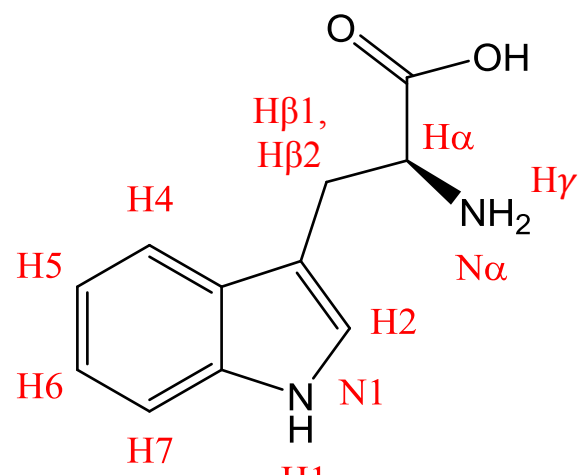

H1

Figure S.3: Nucleus labels of Trp radical in DFT calculations.

\begin{tabular}{|c|c|c|c|c|c|}
\hline \multirow[t]{2}{*}{ Nucleus } & \multicolumn{3}{|c|}{$\mathbf{A} / \mathrm{mT}$} & \multirow[t]{2}{*}{$a_{\text {iso }} / \mathrm{mT}$} & \multirow{2}{*}{$\begin{array}{c}T_{q q} / \mathrm{mT} \\
0.7596\end{array}$} \\
\hline & -0.0336 & 0.0924 & -0.1354 & & \\
\hline \multirow[t]{3}{*}{ N1 } & 0.0924 & 0.3303 & -0.5318 & 0.3215 & -0.3745 \\
\hline & -0.1354 & -0.5318 & 0.6680 & & -0.3851 \\
\hline & -0.9920 & -0.2091 & -0.2003 & & 0.5914 \\
\hline \multirow[t]{3}{*}{$\mathrm{H} 1$} & -0.2091 & -0.2631 & 0.2803 & -0.5983 & -0.1071 \\
\hline & -0.2003 & 0.2803 & -0.5398 & & -0.4843 \\
\hline & -0.2843 & 0.1757 & 0.1525 & & 0.2855 \\
\hline \multirow[t]{2}{*}{$\mathrm{H} 2$} & 0.1757 & -0.2798 & 0.0975 & -0.2780 & -0.0919 \\
\hline & 0.1525 & 0.0975 & -0.2699 & & -0.1936 \\
\hline
\end{tabular}




\begin{tabular}{|c|c|c|c|c|c|}
\hline \multirow{3}{*}{$\mathrm{H} 4$} & -0.5596 & -0.1956 & -0.1657 & \multirow{3}{*}{-0.4880} & 0.3001 \\
\hline & -0.1956 & -0.4020 & 0.0762 & & -0.0480 \\
\hline & -0.1657 & 0.0762 & -0.5021 & & -0.2520 \\
\hline \multirow{3}{*}{ H6 } & -0.0506 & 0.0622 & 0.0889 & \multirow{3}{*}{-0.2083} & 0.1979 \\
\hline & 0.0622 & -0.3100 & -0.0297 & & -0.0494 \\
\hline & 0.0889 & -0.0297 & 0.2642 & & -0.1485 \\
\hline \multirow{3}{*}{$\mathrm{H} 7$} & -0.4355 & -0.1541 & -0.1239 & \multirow{3}{*}{-0.3636} & 0.2540 \\
\hline & -0.1541 & -0.2777 & 0.0864 & & -0.0594 \\
\hline & -0.1239 & 0.0864 & -0.3770 & & -0.1945 \\
\hline \multirow{3}{*}{$\mathrm{H} \beta 1$} & 1.5808 & -0.0453 & -0.0506 & \multirow{3}{*}{1.6046} & 0.1521 \\
\hline & -0.0453 & 1.5575 & 0.0988 & & -0.0456 \\
\hline & -0.0506 & 0.0988 & 1.6752 & & -0.1065 \\
\hline \multirow{3}{*}{$\mathrm{H} \alpha$} & -0.0601 & 0.0037 & 0.0331 & \multirow{3}{*}{-0.0931} & -0.1092 \\
\hline & 0.0037 & -0.0251 & 0.0111 & & 0.0395 \\
\hline & 0.0331 & 0.0111 & -0.1940 & & 0.0698 \\
\hline \multirow{3}{*}{$\mathrm{N} \alpha$} & 0.1295 & -0.0134 & 0.0075 & \multirow{3}{*}{0.1465} & -0.0224 \\
\hline & -0.0134 & 0.1729 & -0.0249 & & -0.0270 \\
\hline & 0.0075 & -0.0249 & 0.1371 & & 0.0431 \\
\hline \multirow{3}{*}{$\mathrm{H} \beta 2$} & 0.1634 & -0.0230 & -0.0064 & \multirow{3}{*}{0.0457} & -0.0758 \\
\hline & -0.0230 & -0.0082 & 0.0158 & & -0.0454 \\
\hline & -0.0064 & 0.0158 & -0.0182 & & 0.1211 \\
\hline \multirow{3}{*}{ H5 } & 0.0051 & 0.0616 & 0.0694 & \multirow{3}{*}{-0.0400} & -0.0632 \\
\hline & 0.0616 & -0.0665 & 0.0391 & & -0.0616 \\
\hline & 0.0694 & 0.0391 & -0.0586 & & 0.1248 \\
\hline
\end{tabular}




\begin{tabular}{|c|c|c|c|c|c|}
\hline \multirow{3}{*}{$\mathrm{H} \gamma$} & 0.0329 & -0.0321 & 0.0587 & \multirow{3}{*}{0.0233} & -0.0617 \\
\hline & -0.0321 & 0.0253 & -0.0169 & & -0.0141 \\
\hline & 0.0587 & -0.0169 & 0.0117 & & 0.0758 \\
\hline
\end{tabular}

Table 2: Hyperfine tensors $(\mathbf{A})$ for the $\operatorname{TrpH}^{\bullet+}$ radical in the same axis system as above [10]. The isotropic $\left(a_{\text {iso }} / \mathrm{mT}\right)$ and anisotropic $\left(T_{q q} / \mathrm{mT}\right)$ interactions are given. 


\section{S. IV. C. Ascorbate Radical}

The hyperfine tensors of the ascorbate radical are taken to be the experimental values given in [13]. The ascorbate radical is assumed to be freely tumbling in vivo such that the hyperfine interactions are rendered isotropic.

\begin{tabular}{|c|c|c|c|c|c|}
\hline \multirow[t]{2}{*}{ Nucleus } & \multicolumn{3}{|c|}{$\mathbf{A} / \mathrm{mT}$} & \multirow[t]{2}{*}{$a_{\text {iso }} / \mathrm{mT}$} & \multirow{2}{*}{$\frac{T_{q q} / \mathrm{mT}}{0.0}$} \\
\hline & 0.176 & 0.0 & 0.0 & & \\
\hline \multirow[t]{3}{*}{$\mathrm{H} 4$} & 0.0 & 0.176 & 0.0 & 0.176 & 0.0 \\
\hline & 0.0 & 0.0 & 0.176 & & 0.0 \\
\hline & 0.019 & 0.0 & 0.0 & & 0.0 \\
\hline \multirow[t]{3}{*}{$\mathrm{H} 6(\times 2)$} & 0.0 & 0.019 & 0.0 & 0.019 & 0.0 \\
\hline & 0.0 & 0.0 & 0.019 & & 0.0 \\
\hline & 0.007 & 0.0 & 0.0 & & 0.0 \\
\hline \multirow[t]{2}{*}{ H5 } & 0.0 & 0.007 & 0.0 & 0.007 & 0.0 \\
\hline & 0.0 & 0.0 & 0.007 & & 0.0 \\
\hline
\end{tabular}

Table 3: Hyperfine tensors $(\mathbf{A})$ for the $\mathrm{Asc}^{\bullet-}$ radical. The isotropic $\left(a_{\mathrm{iso}} / \mathrm{mT}\right)$ and anisotropic $\left(T_{q q} / \mathrm{mT}\right)$ interactions are given. 


\section{References}

[1] A. F. Voter, "Introduction to the kinetic Monte Carlo method," in Radiation effects in solids (K. E. Sickafus, E. A. Kotomin, and B. P. Uberuaga, eds.), pp. 1-23, Springer, Dordrecht, 2007.

[2] M. B. Wilk and R. Gnanadesikan, "Probability plotting methods for the analysis for the analysis of data," Biometrika, vol. 55, no. 1, pp. 1-17, 1968.

[3] D. P. Bertsekas and J. N. Tsitsiklis, Introduction to probability, vol. 1. Athena Scientific, Belmont, MA, 2002.

[4] T. M. Cover and J. A. Thomas, Elements of information theory. John Wiley \& Sons, Hoboken, 2012.

[5] J. C. S. Lau, C. T. Rodgers, and P. J. Hore, "Compass magnetoreception in birds arising from photo-induced radical pairs in rotationally disordered cryptochromes," J. R. Soc. Interface, vol. 9, pp. 3329-3337, 2012.

[6] I. A. Solov'yov, H. Mouritsen, and K. Schulten, "Acuity of a cryptochrome and visionbased magnetoreception system in birds," Biophys. J., vol. 99, no. 1, pp. 40-49, 2010.

[7] T. Ritz, M. Ahmad, H. Mouritsen, R. Wiltschko, and W. Wiltschko, "Photoreceptorbased magnetoreception: optimal design of receptor molecules, cells, and neuronal processing," J. R. Soc. Interface, vol. 7, no. 2, pp. S135-S146, 2010.

[8] M. Frisch, G. Trucks, H. Schlegel, G. Scuseria, M. Robb, J. Cheeseman, J. Montgomery Jr, T. Vreven, K. Kudin, J. Burant, et al., "Gaussian 03, revision C. 02; Gaussian, Inc.: Wallingford, CT, 2004."

[9] C. T. Rodgers, Magnetic Field Effects in Chemical Systems. PhD thesis, University of Oxford, 2007. 
[10] A. A. Lee, J. C. S. Lau, H. J. Hogben, T. Biskup, D. R. Kattnig, and P. J. Hore, "Alternative radical pairs for cryptochrome-based magnetoreception," J. R. Soc. Interface, vol. 11, no. 95, 20131063, 2014.

[11] B. D. Zoltowski, A. T. Vaidya, D. Top, J. Widom, M. W. Young, and B. R. Crane, "Structure of full-length Drosophila cryptochrome," Nature, vol. 480, no. 7377, p. 396, 2011.

[12] C. Levy, B. D. Zoltowski, A. R. Jones, A. T. Vaidya, D. Top, J. Widom, M. W. Young, N. S. Scrutton, B. R. Crane, and D. Leys, "Updated structure of Drosophila cryptochrome," Nature, vol. 495, no. 7441, p. E3, 2013.

[13] G. P. Laroff, R. W. Fessenden, and R. H. Schuler, "Electron spin resonance spectra of radical intermediates in the oxidation of ascorbic acid and related substances," JACS, vol. 94, no. 26, pp. 9062-9073, 1972. 